\title{
Genome-Wide Expression Profiling of Small RNAs in Indian Strain of Rhizoctonia solani AG1-1A Reveals Differential Regulation of milRNAs during Pathogenesis and Crosstalk of Gene Regulation
}

\author{
Naresh Babu Prathi 1,2,+ ${ }^{+}$Chagamreddy Venkata Durga Rani ${ }^{1}$, Sena Munuswamy Balachandran ${ }^{2}$, \\ Vellaisamy Prakasam ${ }^{2}$, Yeshala Chandra Mohan ${ }^{1}$, Sanivarapu Nagalakshmi ${ }^{2}$, Sunil K. Srivastava ${ }^{3}$, \\ Raman Meenakshi Sundaram ${ }^{2}$ and Satendra K. Mangrauthia ${ }^{2, *,+}$ (D)
}

1 Institute of Biotechnology, Professor Jayashankar Telangana State Agricultural University (PJTSAU), Rajendranagar, Hyderabad 500030, India; nareshseshu0962@gmail.com (N.B.P.); ranivenkata@yahoo.com (C.V.D.R.); drycmohan@gmail.com (Y.C.M.)

2 Indian Council of Agricultural Research (ICAR)-Indian Institute of Rice Research, Hyderabad 500030, India; balasena@yahoo.com (S.M.B.); vprakasam.iari@gmail.com (V.P.); nagalakshmi.s9@gmail.com (S.N.); r.sundaram@icar.gov.in (R.M.S.)

check for updates

Citation: Prathi, N.B.; Durga Rani, C.V.; Balachandran, S.M.; Prakasam, V.; Chandra Mohan, Y.; Nagalakshmi, S.; Srivastava, S.K.; Sundaram, R.M.; Mangrauthia, S.K. Genome-Wide Expression Profiling of Small RNAs in Indian Strain of Rhizoctonia solani AG1-1A Reveals Differential Regulation of milRNAs during Pathogenesis and Crosstalk of Gene Regulation. J. Fungi 2021, 7, 561. https://doi.org/10.3390/jof7070561

Academic Editors: Nuria Ferrol and Concepción Azcón Aguilar

Received: 30 April 2021

Accepted: 8 July 2021

Published: 14 July 2021

Publisher's Note: MDPI stays neutral with regard to jurisdictional claims in published maps and institutional affiliations.

Copyright: (c) 2021 by the authors. Licensee MDPI, Basel, Switzerland. This article is an open access article distributed under the terms and conditions of the Creative Commons Attribution (CC BY) license (https:// creativecommons.org/licenses/by/ $4.0 /)$.
3 Department of Microbiology, Swami Shraddhanand College, University of Delhi, Alipur, Delhi 110036, India; sun27sri@gmail.com

* Correspondence: Satendra.KM@icar.gov.in or skmdrr@gmail.com; Tel.: +91-40-24591342

+ Contributed equally.

Abstract: Rhizoctonia solani AG1-1A is a necrotrophic fungus that causes sheath blight disease in rice. The reliable resistant source against this phytopathogenic fungus is not available in the gene pool of rice. Better understanding of pathogen genomics and gene regulatory networks are critical to devise alternate strategies for developing resistance against this noxious pathogen. In this study, miRNA-like RNAs (milRNAs) of an Indian strain of $R$. solani were identified by deep sequencing of small RNAs. We identified 128 known and 22 novel milRNAs from 20,963,123 sequence reads. These milRNAs showed 1725 target genes in the fungal genome which include genes associated with growth, development, pathogenesis and virulence of $R$. solani. Notably, these fungal milRNAs showed their target genes in host (rice) genome also which were later verified by qRT-PCR. The host target genes are associated with auxin metabolism, hypersensitive response, defense genes, and genes related to growth and development of rice. Osa-vacuolar-sorting receptor precursor: Rhi-milR-13, Osa-KANADI1:Rhi-milR-124, Osa-isoflavone reductase: Rhi-milR-135, Osa-nuclear transcription factor Y:Rhi-milR-131, Osa-NB-ARC domain containing protein: Rhi-milR-18, and Osa-OsFBX438: Rhi-milR-142 are notable potential regulons of host target genes: fungal milRNAs that need to be investigated for better understanding of the crosstalk of RNAi pathways between $R$. solani and rice. The detailed expression analysis of 17 milRNAs by qRT-PCR was analysed during infection at different time points of inoculation, at different growth stages of the host, in four different genotypes of the host, and also in four different strains of fungi which revealed differential regulation of milRNAs associated with pathogenesis and virulence. This study highlights several important findings on fungal milRNAs which need to be further studied and characterized to decipher the gene expression and regulation of this economically important phytopathogen.

Keywords: microRNA; sheath blight; Oryzasativa; fungi; strain; qRT-PCR

\section{Introduction}

Rhizoctonia solani AG1-1AKühn (Teleomorph: Thanatephorus cucumeris (A.B. Frank) Donk.) is a basidiomycete semi-saprotrophic soil-borne fungal pathogen that causes sheath blight disease in rice. In Eastern Asia, R. solani causes a loss of 6 million tons of rice 
grains per year [1]. India suffers $10 \%$ yield loss in rice due to sheath blight disease every year [2]. The pathogen is present in the form of sclerotia or mycelium, and severely affects high yielding, semi-dwarf, and nitrogen-responsive rice cultivars. Initial symptoms are noticed on leaf sheaths near water level. Warm and humid weather, dense planting, and high nitrogen inputs are the favourable conditions for its aggravation. Until now, rice germplasm immune to $R$. solani has not been known. Among several other factors, poor understanding of pathogen biology is one of the key factors that hamper the development of resistant cultivars either through breeding or transgenic approaches. Recently, efforts have been made to understand the regulation of the genome and transcriptome of $R$. solan $i$ during pathogenesis [3-5].

Clear understanding of pathogen genomics and gene regulatory networks is extremely important for drawing effective strategies for resistance development against diseases. One such example is the rice-Xanthomonas oryzae pv. oryzae (Xoo) pathosystem where deeper understanding of bacterial TALE (transcription activator-like effector) genes and their interaction with rice SWEET genes has helped developing durable and broad spectrum bacterial blight-resistant rice genotypes through marker-assisted breeding and genome editing [6-9]. Genomics has been extremely useful to facilitate host-induced gene silencing (HIGS) technology for the control of Fusarium diseases through targeted disruption of key genes [10]. Recently, Dong and Ronald (2019) [11] presented comprehensive information on pathogen genes used for disease resistance development through genetic engineering, RNAi, and genome editing. In addition to genetic resistance, fungal genomics is crucial for fungicide development by identifying potential fungicide targets, their validation and mode of action, as demonstrated in several pathosystems [12]. For more than a decade, small RNAs have taken central stage in the area of genomics due to their versatile functions in gene regulation and metabolic pathways $[13,14]$.

Small RNAs (sRNAs) of $<200$ nucleotides are classified into different categories such as miRNA, siRNA, piwiRNA, snoRNA and t-RNAs etc. Recently, more sRNAs have been discovered based on their site of synthesis, binding proteins, and secondary structure and function, for example, trans-acting siRNAs (tasiRNAs) [15], repeat associated siRNAs (rasiRNAs) [16], heterochromatic small RNAs (hcRNAs) [17], and small scan RNAs (scnRNAs) [18] etc. MicroRNAs (miRNAs) are 21-24 nucleotide endogenous non-coding RNA molecules that work as defensive regulators in eukaryotes through post-transcriptional gene regulation. To date, numerous miRNAs have been discovered in plants and animals, but very few miRNAs have been reported in fungi [19]. Recent advancement in sequencing technologies and bioinformatics tools facilitated the identification of miRNAs in fungi [19-21]. MicroRNAs are critical regulators of host-pathogen interaction, and may help disease initiation and establishment [19,21-23].

In the model fungi Neurospora crassa, four different types of miRNA-like RNAs (milRNAs) processed through a dicer-independent pathway were identified. The proteins responsible for the production of these milRNAs are different from the plants and animals to a large extent [24]. Considering the essential functions of miRNAs in gene regulation and metabolic processes, there is a necessity to study the fungal genome encoded milRNAs which would give different insights and roles of microRNAs in pathogenesis and disease development. To date, very few milRNAs have been reported in plant pathogenic fungi, which could be due to the low accumulation of milRNAs or the small number of cells at certain infection stages, and also due to lesser attention of researchers. Recently, Meng et al. (2021) [25] identified virulence-associated milRNAs from R. solani AG1-IA infecting maize crop. With this perspective, our current study focussed on discovering the milRNAs in $R$. solani causing sheath blight disease in rice. Genome-wide small RNAs isolated from a pure culture of the Indian strain of $R$. solani AG1-1Awere sequenced to identify the known and novel milRNAs. Later, the targets of these milRNAs were predicted in $R$. solani and its host (rice) genome. Expression of 17 fungal milRNAs was analyzed in infected tissue of rice at different time points of inoculation, in different genotypes and growth stages of rice, and 
also in different strains of fungi. Expression analysis of target genes present in fungal and rice genome suggested that milRNAs are critical regulators of host-pathogen interaction.

\section{Material and Methods}

\subsection{Fungal and Plant Material}

In this study, four strains of R. solani AG1-IA were used. These strains were Wgl-2, Chn-1, Imph-2 and Lud-1 [26]. Four rice genotypes as a fungal host were used that include susceptible (TN1 and BPT5204) and resistant (Tetep and Pankaj) cultivars.

\subsection{RNA Isolation and Sequencing}

The highly virulent Indian strain of $R$. solani AG1-IA Wgl-2 was grown in growth medium having pectin as a carbon source. The fungal sclerotium was placed in liquid medium $\left(0.7 \mathrm{~g} \mathrm{~K}_{2} \mathrm{HPO}_{4}, 0.5 \mathrm{~g} \mathrm{KH}_{2} \mathrm{PO}_{4}, 0.5 \mathrm{~g} \mathrm{MgSO}_{4}, 0.01 \mathrm{~g} \mathrm{FeSO}_{4}, 0.001 \mathrm{~g} \mathrm{ZnSO}\right.$, and $10 \mathrm{~g}$ pectin in $1 \mathrm{~L}$ distilled and autoclaved water) at $28^{\circ} \mathrm{C}$ for $48-72 \mathrm{~h}$ as a still culture. The mycelium from three biological replicates was harvested and pooled to extract the RNA using TRIzol reagent (Invitrogen, Waltham, MA, USA). The quality of RNA was checked by Nanodrop (Thermo Fisher Scientific, Waltham, MA, USA), Bioanalyzer (Agilent 2100), and agarose gel electrophoresis to check the integrity, concentration, and contamination. After quality control (QC), the sequencing library was constructed by TruSeq Small RNA Library Preparation Kit (Illumina, CA, USA). The cDNA library was prepared by sequencing adaptor ligation, reverse transcription, PCR enrichment, purification and size selection. The QC of library was analyzed by Qubit 2.0 (preliminary library concentration), Agilent 2100 (insert size), and qPCR (effective concentration of library). The QC passed libraries were fed into HiSeq 2500 sequencer for sequencing.

\section{3. milRNAs Identification in R. solani}

The sequence data were analyzed to identify the milRNAs in the R. solani genome. Clean reads without adaptorcontamination were mapped to reference genome available in the RSIADB database [27]. Further non-coding sRNAs like rRNA, snRNA, snoRNA, and tRNAs were removed by using the Rfamdatabase (https:/ / rfam.xfam.org/, accessed on 1 July 2017). The mRNA sequences or coding transcripts were filtered using RSIADB database. Later, miRNA prediction was undertaken using miREAP and miRDeep2 software for identification of both known and novel milRNAs. The secondary structures of these potential novel milRNA precursors were predicted by the RNAfoldWebServer program (http:/ / rna.tbi.univie.ac.at/cgi-bin/RNAWebSuite/RNAfold.cgi, accessed on 1 July 2017). Putatively novel milRNAs were identified based on the following criteria: (1) mature milRNA sequence should be in either of the arm of hairpin structure, (2) number of mismatches 4 or less, (3) no loop or break in milRNA sequences, (4) asymmetric bulges should be minimal in size (one or two bases) and frequency (typically one or less), especially within the milRNA/milRNA* duplex $[3,20,28]$.

\subsection{The Target Gene Prediction in R. solani and Rice Genome}

The target genes of milRNAs in the R. solani genome were predicted by using Miranda software [21] and the RSIADB database. The target genes of fungal encoded milRNAs in rice genome were predicted by using the psRNA Target database [29]. The default criteria of the target prediction tool were used for identification of milRNA target genes.

\subsection{Expression Analysis of Fungal milRNAs and Their Target Genes}

Forty-five day old plants of rice cultivars TN1, Pankaj, BPT 5204, and Tetep were inoculated by $R$. solani AG1IA Wgl-2 strain. In parallel, TN1 was inoculated by three other strains, i.e., Chn-1, Imph-2 and Lud-1. For inoculation, the fungal sclerotium was placed in the sheath tissue of rice using a transparent cello tape. The sheath tissue was harvested from different rice genotypes (TN1, Pankaj, BPT 5204, and Tetep) infected by the Wgl-2 strain. Also, infected sheath tissue was harvested from TN1 at different time 
points of infection ( $18 \mathrm{~h}, 24 \mathrm{~h}, 48 \mathrm{~h}, 72 \mathrm{~h}, 96 \mathrm{~h}$, and 5 days after inoculation) as well as from 45- (vegetative) and 80- (reproductive) day-old growth stages infected by the Wgl-2 strain. To analyze the strain specific milRNAs regulation, tissue was harvested from TN1 infected by three other strains (Chn-1, Imph-2, and Lud-1). Small RNAs and mRNAs were isolated from fungal infected rice tissue and pure culture of fungi (grown in potato dextrose agar medium) using the mirVana miRNA isolation kit (Ambion, TX, USA) according to the manufacturer's instructions. cDNA synthesis of normalized sRNAs and mRNAs was performed using the miScript II RT kit (Qiagen, Hilden, Germany) and Improm-II reverse transcription system (Promega, Madison, WI, USA), respectively. The respective cDNA was used as template for milRNA and target gene quantification using miScript SYBR Green PCR kit (Qiagen, Germany) and SYBR Premix Ex-Taq (Takara, Shiga, Japan), respectively. Three biological replicates and two technical replicates were used for all the qRT-PCR experiments. Following the manufacturer instructions, all the kit reagents along with the milRNA and gene-specific primers (Table S1) were used for setting up the qRT-PCR reaction in a CFX96 Real-Time System (BIO-RAD, Hercules, CA, USA). The temperature profile was followed as described in previous report [30]. To ensure specificity of amplified product, melt curve analysis and agarose gel electrophoresis were used. To quantify the relative expression of milRNAs and target genes, the comparative threshold cycle $\left(C_{T}\right)$ method was followed. The relative expression was calculated from $2^{-\Delta \Delta \mathrm{Ct}}$. The standard error was calculated as reported previously [31].

\section{Results}

\subsection{Sequence Statistics}

The milRNAs encoded by the R. solani AG1 IA genome were identified through Illumina deep sequencing of small RNAs. All the sequence reads were aligned to the reference genome of $R$. solani available in the RSIADB database [27] and the mapped clean reads were further used to identify the known and novel milRNAs. After deep sequencing of $R$. solani small RNAs 20,963,123 raw reads and 18,084,903 clean reads were obtained. The clean reads were used to filter 1,231,411 reads of 18-24 nts size of which 185,760 were unique reads. 2574 reads of $R$. solani aligned with hairpin sequences of known milRNAs [21], while 619,582 reads were utilized for prediction of novel milRNAs.

\subsection{Identification of Known and Novel milRNAs}

128 known milRNAs of $R$. solani were identified in Indian strain of the pathogen (Table S2). Among these, Rhi-milR-16, Rhi-milR-22, Rhi-milR-35, Rhi-milR-57, Rhi-milR-91, and Rhi-milR-112 showed higher read count than the others. Twenty two novel milRNAs were predicted among which scaffold13_22000, scaffold47_37005, scaffold47_37523, scaffold47_37559, scaffold47_37341, scaffold47_37407, and scaffold47_36418 showed higher read account than others. Notably, scaffold13_22000 showed $>6000$ read count (Table 1). The secondary structure of a few representative novel milRNAs is shown in Figure 1. 
Table 1. Novel milRNAs predicted from small RNA sequence data of Rhizoctonia solani AG1-1A Wgl-2 strain.

\begin{tabular}{|c|c|c|c|c|c|c|}
\hline S. No. & Novel milRNA & miRDeep2 Score & $\begin{array}{c}\text { Estimated Probability That } \\
\text { the miRNA Candidate Is a } \\
\text { True Positive }\end{array}$ & Total Read Count & Consensus Mature Sequence & Precursor Coordinate \\
\hline 1 & scaffold47_37407 & 193.1 & $70+/-24 \%$ & 379 & aaucccuaggaucuccacuug & scaffold47:62019..62062:- \\
\hline 2 & scaffold47_37523 & 193 & $70+/-24 \%$ & 379 & aaucccuaggaucuccacuug & scaffold47:127383..127426:- \\
\hline 3 & scaffold47_37341 & 192.9 & $70+/-24 \%$ & 379 & aaucccuaggaucuccacuug & scaffold47:26292..26335:- \\
\hline 4 & scaffold47_36931 & 30.3 & $70+/-24 \%$ & 80 & Uggacaauguucugugggguu & scaffold47:118388..118435:+ \\
\hline 5 & scaffold145_44745 & 17.3 & $70+/-24 \%$ & 37 & Gacuaaucagcguugggcgcauuu & scaffold145:16023..16102:+ \\
\hline 6 & scaffold6_14191 & 6 & $66+/-23 \%$ & 11 & Ugggaagggugcuagggaccucu & scaffold6:504695..504742:- \\
\hline 7 & scaffold10_19054 & 1.3 & $35+/-26 \%$ & 3 & Ugccgacugugcuccegccaucg & scaffold10:751369..751434:- \\
\hline 8 & scaffold1_2221 & 0.9 & $16+/-15 \%$ & 5 & Cagcacacuggaccgagagcucu & scaffold1:3047379..3047427:+ \\
\hline 9 & scaffold47_37005 & 0.8 & $16+/-15 \%$ & 249 & Agauuuuagggaccccguaacuc & scaffold47:124971..125020:+ \\
\hline 10 & scaffold12_20737 & 0.8 & $16+/-15 \%$ & 27 & gaguuuuggacuggcaccc & scaffold12:518031..518072:+ \\
\hline 11 & scaffold7_15416 & 0.6 & $16+/-15 \%$ & 2 & uguuggcgugcugcugaaccg & scaffold7:443987..444035:- \\
\hline 12 & scaffold47_35943 & 0.4 & $16+/-15 \%$ & 29 & Aaggaaauucucguagggcuucu & scaffold47:16996..17045:+ \\
\hline 13 & scaffold47_36418 & 0.4 & $16+/-15 \%$ & 94 & Uuucuauggaccaccacgaguacu & scaffold47:65345..65392:+ \\
\hline 14 & scaffold49_37930 & 0.4 & $16+/-15 \%$ & 2 & Ccuuugcuaggcuccugcgcgcau & scaffold49:58540..58612:+ \\
\hline 15 & scaffold48_37687 & 0.3 & $16+/-15 \%$ & 14 & Ucucgaagcgcgacucuguccuu & scaffold48:142724..142771:+ \\
\hline 16 & scaffold30_30807 & 0.3 & $16+/-15 \%$ & 30 & Agcgcaacucgaccucugaucacg & scaffold30:89871..89933:- \\
\hline 17 & scaffold34_32450 & 0.2 & $16+/-15 \%$ & 52 & Aucgcugacugcgguguccucu & scaffold34:218833..218913:- \\
\hline 18 & scaffold13_22000 & 0 & $16+/-15 \%$ & 6028 & Aaggugccggaauauacgcucau & scaffold13:463575..463652:- \\
\hline 19 & scaffold130_44481 & 0 & $16+/-15 \%$ & 2 & ccgugcaacggacgaucgac & scaffold130:10887..10931:- \\
\hline 20 & scaffold47_37559 & 0 & $16+/-15 \%$ & 148 & Cguggacgggccgcauccc & scaffold47:139094..139138:- \\
\hline 21 & scaffold22_27474 & 0 & $16+/-15 \%$ & 2 & Ucgggcgagacgagugcuuucc & scaffold22:336752..336810:+ \\
\hline 22 & scaffold1_2114 & 0 & $16+/-15 \%$ & 3 & Ccugucgcugcucgugaagccucu & scaffold1:2908975..2909036:+ \\
\hline
\end{tabular}



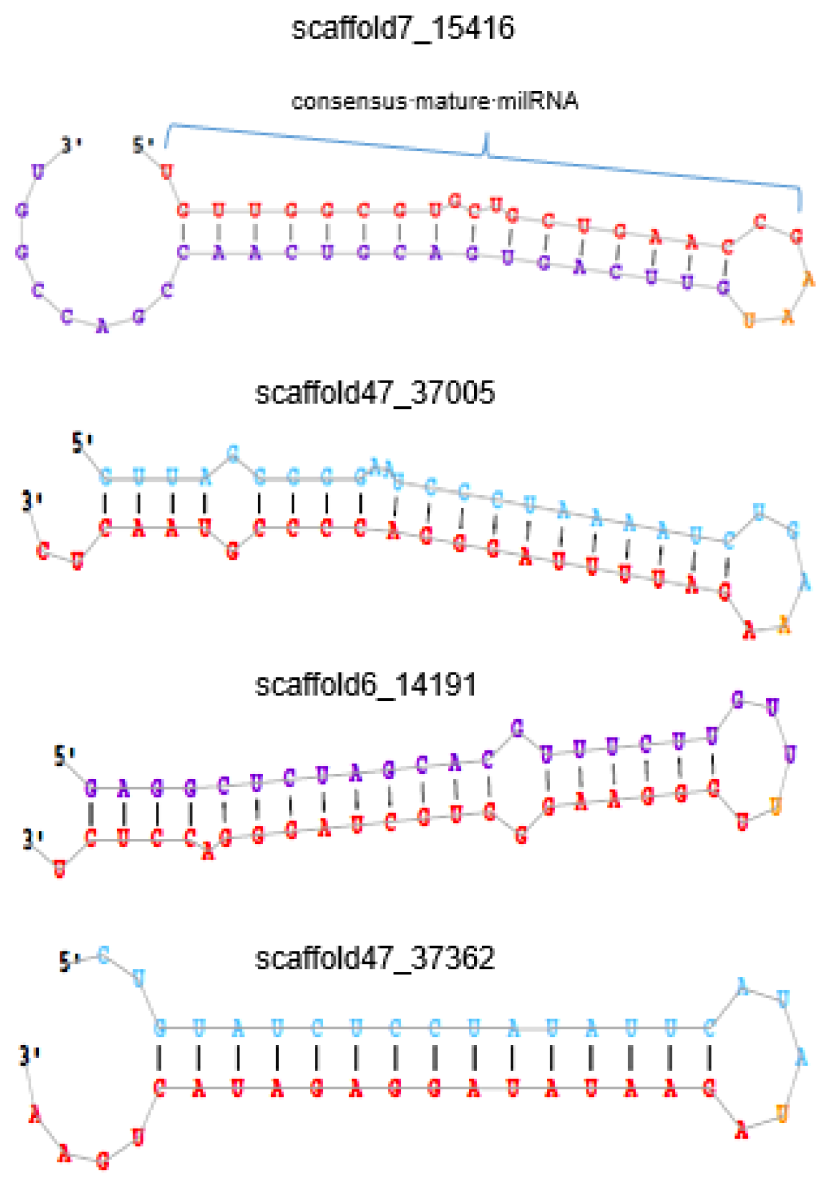

Scaffold47_36068

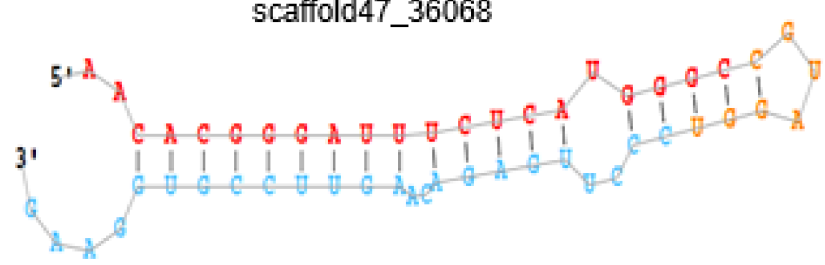

scaffold49_37930

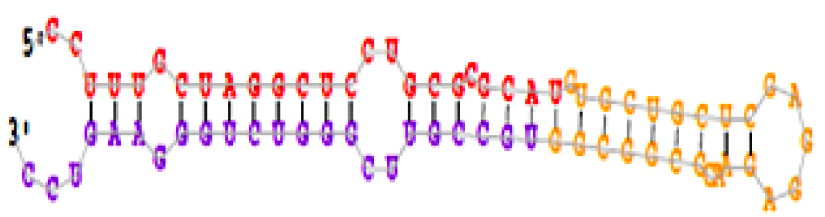

Figure 1. Depiction of representative novel milRNAs identified in this study. Red colour nucleotides indicate the sequence of mature milRNAs. Blue, purple, and golden colours are the backbone of novel milRNAs.

\subsection{Identification of Target Genes in R. solani Genome}

We identified 1725 target genes of 128 known and 22 novel milRNAs in the $R$. solani genome (Table S3). The known milRNAs targeting the genes encoding carbohydrate active enzymes, secretory proteins, and other critical proteins associated with fungal biology are listed (Tables 2 and S4). Also, the target genes of novel milRNAs were predicted (Table 3). Notably, Scaffold47_36931 showed the highest number (12) of target genes. Rhi-milR-141, Rhi-milR-120, Rhi-milR-41, and Rhi-milR-91 showed pectin esterase and pectate lyase domain-containing protein genes as their targets. 
Table 2. Gene targets of Rhizoctonia solani-milRNAs. These target genes encode carbohydrate active enzymes and secretory proteins of fungus.

\begin{tabular}{|c|c|c|}
\hline milRNA Id & Target Gene & Gene Description \\
\hline \multicolumn{3}{|r|}{ Carbohydrate Active Enzymes } \\
\hline Rhi-milR-56 & AG1IA_01218 & Beta-glucosidase (EC 3.2.1.21) \\
\hline Rhi-milR-120 & AG1IA_01405 & 1,3-beta-glucan synthase component GLS2 \\
\hline Rhi-milR-122 & AG1IA_01406 & Sterol 3-beta-glucosyltransferase (EC 2.4.1.173) (Autophagy-related protein 26) \\
\hline Rhi-milR-91 & AG1IA_02027 & Endoplasmic reticulum protein \\
\hline Rhi-milR-169 & AG1IA_02441 & Beta-xylosidase \\
\hline Rhi-milR-146 & AG1IA_02513 & Chitin deacetylase \\
\hline Rhi-milR-120 & AG1IA_02835 & $\begin{array}{l}\text { Putative 1,4-alpha-glucan branching enzyme from glycoside } \\
\text { hydrolase family GH13 }\end{array}$ \\
\hline Rhi-milR-141 & AG1IA_02889 & Pectinesterase (EC 3.1.1.11) \\
\hline Rhi-milR-150 & AG1IA_03463 & Glycosyltransferase family 2 protein \\
\hline Rhi-milR-36 & AG1IA_03939 & Beta-mannosidase \\
\hline Rhi-milR-91 & AG1IA_04214 & Trehalose 6-phosphate phosphatase, glycosyltransferase family 20 protein \\
\hline Rhi-milR-81 & AG1IA_04527 & Uridine Di Phosphate- $N$-acetylglucosaminyltransferase \\
\hline Rhi-milR-68 & AG1IA_04727 & Farnesyltransferase subunit beta \\
\hline Rhi-milR-111 & AG1IA_04740 & Polysaccharide lyase family 1 protein \\
\hline Rhi-milR-27 & AG1IA_04862 & 1,3-beta-glucan synthase component GLS2 \\
\hline Rhi-milR-45 & AG1IA_05653 & Glycoside hydrolase family 51 protein \\
\hline Rhi-milR-52 & AG1IA_05719 & Chitin synthase D \\
\hline Rhi-milR-119 & AG1IA_05754 & Glycogen phosphorylase \\
\hline Rhi-milR-42 & AG1IA_05803 & Alpha-galactosidase (EC 3.2.1.22) (Melibiase) \\
\hline Rhi-milR-139 & AG1IA_05807 & Adenylosuccinate synthetase (AMPSase) (AdSS) (EC 6.3.4.4) (IMP-aspartate ligase) \\
\hline Rhi-milR-122 & AG1IA_06014 & Glycoside hydrolase family 51 protein \\
\hline Rhi-milR-41 & AG1IA_06294 & Glycoside hydrolase family 3 protein \\
\hline Rhi-milR-58 & AG1IA_06593 & Alpha glucosidase II, alpha subunit, putative \\
\hline Rhi-milR-167 & AG1IA_07255 & Killer toxin alpha/beta \\
\hline Rhi-milR-51 & AG1IA_07341 & Galactan 1,3-beta-galactosidase \\
\hline Rhi-milR-120 & AG1IA_07743 & Pectinesterase (EC 3.1.1.11) \\
\hline Rhi-milR-162 & AG1IA_07787 & Glycoside hydrolase family 31 protein \\
\hline Rhi-milR-168 & AG1IA_07905 & Glycoside hydrolase family 95 protein \\
\hline Rhi-milR-97 & AG1IA_08771 & Exo-beta-1,3-glucanase \\
\hline \multicolumn{3}{|r|}{ Secretory Proteins } \\
\hline Rhi-milR-150 & AG1IA_00157 & Polysaccharide deacetylase domain-containing protein \\
\hline Rhi-milR-98 & AG1IA_01858 & Uncharacterized protein \\
\hline Rhi-milR-124 & AG1IA_01958 & Rad1 domain-containing protein \\
\hline Rhi-milR-95 & AG1IA_02532 & Lipase domain-containing protein \\
\hline Rhi-milR-144 & AG1IA_03100 & Glycosyl hydrolase family 61 domain-containing protein \\
\hline Rhi-milR-122 & AG1IA_03118 & Uncharacterized protein \\
\hline Rhi-milR-141 & AG1IA_03171 & Copper/zinc superoxide dismutase domain-containing protein \\
\hline Rhi-milR-165 & AG1IA_05741 & Uncharacterized protein \\
\hline
\end{tabular}


Table 2. Cont.

\begin{tabular}{ccc}
\hline milRNA Id & Target Gene & Gene Description \\
\hline Rhi-milR-141 & AG1IA_06494 & Uncharacterized protein \\
\hline Rhi-milR-122 & AG1IA_07117 & Uncharacterized protein \\
\hline Rhi-milR-52 & AG1IA_07216 & Uncharacterized protein \\
\hline Rhi-milR-19 & AG1IA_07698 & Tyrosinase domain-containing protein \\
\hline Rhi-milR-54 & AG1IA_08056 & Uncharacterized protein \\
\hline Rhi-milR-144 & AG1IA_08227 & Cytochrome P450 domain-containing protein \\
\hline Rhi-milR-90 & AG1IA_08293 & Uncharacterized protein \\
\hline Rhi-milR-146 & AG1IA_08653 & Uncharacterized protein \\
\hline Rhi-milR-122 & AG1IA_08711 & Protein tyrosine kinase domain-containing protein \\
\hline Rhi-milR-144 & AG1IA_09802 & Cytochrome P450 domain-containing protein \\
\hline Rhi-milR-130 & AG1IA_10060 &
\end{tabular}

Table 3. Target genes of Rhizoctonia solani novel milRNAs.

\begin{tabular}{|c|c|c|c|}
\hline S. No. & Novel milRNA & Target Gene & Gene Description \\
\hline 1 & scaffold47_37407 & AG1IA_04067 & Ubiquitin conjugating enzyme family protein \\
\hline 2 & scaffold47_37407 & AG1IA_02707 & MFS transporter, putative \\
\hline 3 & scaffold47_37523 & AG1IA_04067 & Ubiquitin conjugating enzyme family protein \\
\hline 4 & scaffold47_37523 & AG1IA_02707 & MFS transporter, putative \\
\hline 5 & scaffold47_37341 & AG1IA_04067 & Ubiquitin conjugating enzyme family protein \\
\hline 6 & scaffold47_37341 & AG1IA_02707 & MFS transporter, putative \\
\hline 7 & scaffold47_36931 & AG1IA_08169 & Phosphatidylinositol 3-kinase tor2 \\
\hline 8 & scaffold47_36931 & AG1IA_05744 & DNA-directed RNA polymerase subunit (EC 2.7.7.6) \\
\hline 9 & scaffold47_36931 & AG1IA_06882 & EOS1 domain-containing protein \\
\hline 10 & scaffold47_36931 & AG1IA_06165 & ABC transporter \\
\hline 11 & scaffold47_36931 & AG1IA_07629 & Uncharacterized protein \\
\hline 12 & scaffold47_36931 & AG1IA_07663 & ATP-dependent rRNA helicase RRP3 \\
\hline 13 & scaffold47_36931 & AG1IA_04769 & Fungal zn(2)-Cys(6) binuclear cluster domain-containing protein \\
\hline 14 & scaffold47_36931 & AG1IA_08613 & BMR1 protein \\
\hline 15 & scaffold47_36931 & AG1IA_00053 & Uncharacterized protein \\
\hline 16 & scaffold47_36931 & AG1IA_06822 & Uncharacterized protein \\
\hline 17 & scaffold47_36931 & AG1IA_07843 & Uncharacterized protein \\
\hline 18 & scaffold47_36931 & AG1IA_08221 & Molybdenum cofactor biosynthesis protein \\
\hline 19 & scaffold145_44745 & AG1IA_06624 & Uncharacterized protein \\
\hline 20 & scaffold145_44745 & AG1IA_10187 & RNase $\mathrm{H}$ domain-containing protein \\
\hline 21 & scaffold145_44745 & AG1IA_10310 & TFIIA domain-containing protein \\
\hline 22 & scaffold145_44745 & AG1IA_02688 & GPI transamidase component PIG-S \\
\hline 23 & scaffold145_44745 & AG1IA_05493 & Uncharacterized protein \\
\hline 24 & scaffold145_44745 & AG1IA_04447 & HLH domain-containing protein \\
\hline
\end{tabular}

Abbreviations: MFS-Major Facilitator Superfamily, TFIIA-Transcription Factor IIA, GPI—Glycosylphosphatidylinositol, PIG-SPhosphatidylinositol glycan biosynthesis class S protein, HLH-Helix Loop Helix. 


\subsection{Identification of R. solani-milRNAs Target Genes in Oryza Sativa Genome}

In addition to identifying the gene targets of fungal encoded milRNAs in fungal genome, target genes of these milRNAs were also searched in its host genome. Interestingly, 22 known and 2 novel milRNAs showed their target genes in the rice genome. Some of these notable target genes:milRNA regulatory pairs are Osa-KANADI1:Rhi-milR-124, Osa-vacuolar-sorting receptor precursor:Rhi-milR-13, Osa-nuclear transcription factor Y:Rhi-milR-131, Osa-isoflavone reductase: Rhi-milR-135, Osa-OsFBX438: Rhi-milR-142, Osa-NB-ARC domain containing protein: Rhi-milR-18, Osa-plastocyanin-like domain containing protein:Rhi-milR-22, Osa-cytochrome P450 81E1: Rhi-milR-26, Osa-response regulator receiver domain containing protein: Rhi-milR-43, Osa-serine/threonine-protein kinase receptor precursor: Rhi-milR-51, Osa-OsMADS58: Rhi-milR-66, and Osa-extra-large G-protein-related: Rhi-milR-81. Among the novel miRNAs, Scaffold130_44481 milRNA targets the Osa-plastocyanin-like domain containing protein while scaffold47_37559 targets transferase family protein (Table 4).

Table 4. Target genes of Rhizoctonia solani milRNAs in rice genome; 24 milRNAs showed their target as rice genes encoding proteins associated with defense and other metabolic processes.

\begin{tabular}{|c|c|c|c|c|}
\hline milRNAs & Target Gene Accession & Expect & Gene Description & Inhibition \\
\hline Rhi-milR-1 & LOC_Os01g47740.2 & 3 & $\begin{array}{l}\text { cDNA I zinc finger, } \mathrm{C} 3 \mathrm{HC} 4 \text { type domain containing } \\
\text { protein, expressed }\end{array}$ & Translation \\
\hline Rhi-milR-111 & LOC_Os07g48720.3 & 2.5 & cDNA I MAG2, putative, expressed & Cleavage \\
\hline Rhi-milR-124 & LOC_Os09g23200.1 & 2.5 & cDNA I KANADI1, putative, expressed & Cleavage \\
\hline \multirow{2}{*}{ Rhi-milR-13 } & LOC_Os10g20630.1 & 1 & $\begin{array}{l}\text { cDNA | vacuolar-sorting receptor precursor, } \\
\text { putative, expressed }\end{array}$ & Cleavage \\
\hline & LOC_Os11g02464.1 & 2.5 & $\begin{array}{c}\text { cDNA I vacuolar-sorting receptor precursor, } \\
\text { putative, expressed }\end{array}$ & Cleavage \\
\hline Rhi-milR-130 & LOC_Os06g16140.1 & 2.5 & cDNA I expressed protein & Cleavage \\
\hline \multirow{8}{*}{ Rhi-milR-131 } & LOC_Os12g42400.4 & 2.5 & $\begin{array}{l}\text { cDNA I nuclear transcription factor } Y \text { subunit, } \\
\text { putative, expressed }\end{array}$ & Cleavage \\
\hline & LOC_Os12g42400.1 & 2.5 & $\begin{array}{l}\text { cDNA I nuclear transcription factor } Y \text { subunit, } \\
\text { putative, expressed }\end{array}$ & Cleavage \\
\hline & LOC_Os02g58790.5 & 3 & cDNA I cell division inhibitor, putative, expressed & Cleavage \\
\hline & LOC_Os02g58790.2 & 3 & cDNA I cell division inhibitor, putative, expressed & Cleavage \\
\hline & LOC_Os02g58790.1 & 3 & cDNA I cell division inhibitor, putative, expressed & Cleavage \\
\hline & LOC_Os02g58790.4 & 3 & cDNA I cell division inhibitor, putative, expressed & Cleavage \\
\hline & LOC_Os02g58790.3 & 3 & cDNA I cell division inhibitor, putative, expressed & Cleavage \\
\hline & LOC_Os01g55200.1 & 3 & cDNA I potassium channel KAT1, putative, expressed & Cleavage \\
\hline \multirow[b]{2}{*}{ Rhi-milR-135 } & LOC_Os12g16290.1 & 2.5 & cDNA I isoflavone reductase, putative, expressed & Cleavage \\
\hline & LOC_Os05g45180.1 & 3 & $\begin{array}{c}\text { cDNA I anthocyanidin 5,3-O-glucosyltransferase, } \\
\text { putative, expressed }\end{array}$ & Cleavage \\
\hline Rhi-milR-142 & LOC_Os12g03740.1 & 2.5 & $\begin{array}{l}\text { cDNA I OsFBX438-F-box domain containing } \\
\text { protein, expressed }\end{array}$ & Cleavage \\
\hline Rhi-milR-159 & LOC_Os12g43720.1 & 3 & $\begin{array}{l}\text { cDNA l early-responsive to dehydration } \\
\text { protein-related, putative, expressed }\end{array}$ & Cleavage \\
\hline Rhi-milR-160 & LOC_Os03g24410.1 & 1.5 & cDNA I expressed protein & Cleavage \\
\hline \multirow{3}{*}{ Rhi-milR-18 } & LOC_Os08g30660.1 & 2.5 & $\begin{array}{l}\text { cDNA I NB-ARC domain containing } \\
\text { protein, expressed }\end{array}$ & Cleavage \\
\hline & LOC_Os12g02570.2 & 2.5 & cDNA I expressed protein & Cleavage \\
\hline & LOC_Os12g02570.1 & 2.5 & cDNA I expressed protein & Cleavage \\
\hline
\end{tabular}


Table 4. Cont

\begin{tabular}{|c|c|c|c|c|}
\hline milRNAs & Target Gene Accession & Expect & Gene Description & Inhibition \\
\hline \multirow{3}{*}{ Rhi-milR-20 } & LOC_Os01g25430.1 & 2 & cDNA I expressed protein & Cleavage \\
\hline & LOC_Os07g48200.2 & 2.5 & $\begin{array}{l}\text { cDNA I B3 DNA binding domain containing protein, } \\
\text { putative, expressed }\end{array}$ & Cleavage \\
\hline & LOC_Os07g48200.1 & 2.5 & $\begin{array}{l}\text { cDNA I B3 DNA binding domain containing protein, } \\
\text { putative, expressed }\end{array}$ & Cleavage \\
\hline Rhi-milR-22 & LOC_Os02g49350.1 & 2.5 & $\begin{array}{l}\text { cDNA I plastocyanin-like domain containing protein, } \\
\text { putative, expressed }\end{array}$ & Cleavage \\
\hline Rhi-milR-26 & LOC_Os03g55250.1 & 2.5 & cDNA I cytochrome P450 81E1, putative, expressed & Cleavage \\
\hline Rhi-milR-43 & LOC_Os04g28160.1 & 3 & $\begin{array}{l}\text { cDNA | response regulator receiver domain } \\
\text { containing protein, expressed }\end{array}$ & Cleavage \\
\hline Rhi-milR-51 & LOC_Os04g34390.1 & 3 & $\begin{array}{l}\text { cDNA I serine/threonine-protein kinase receptor } \\
\text { precursor, putative, expressed }\end{array}$ & Cleavage \\
\hline \multirow{3}{*}{ Rhi-milR-61 } & LOC_Os10g26660.2 & 2.5 & cDNA I expressed protein & Translation \\
\hline & LOC_Os08g40620.1 & 2.5 & $\begin{array}{l}\text { cDNA | rabGAP/TBC domain-containing protein, } \\
\text { putative, expressed }\end{array}$ & Cleavage \\
\hline & LOC_Os08g40620.2 & 2.5 & $\begin{array}{l}\text { cDNA | rabGAP/TBC domain-containing protein, } \\
\text { putative, expressed }\end{array}$ & Cleavage \\
\hline Rhi-milR-66 & LOC_Os05g11414.1 & 2.5 & $\begin{array}{l}\text { cDNA I OsMADS58-MADS-box family gene with } \\
\text { MIKCc type-box, expressed }\end{array}$ & Cleavage \\
\hline \multirow{2}{*}{ Rhi-milR-81 } & LOC_Os05g50910.2 & 3 & $\begin{array}{l}\text { cDNA I extra-large G-protein-related, } \\
\text { putative, expressed }\end{array}$ & Cleavage \\
\hline & LOC_Os05g50910.1 & 3 & $\begin{array}{l}\text { cDNA I extra-large G-protein-related, } \\
\text { putative, expressed }\end{array}$ & Cleavage \\
\hline \multirow{2}{*}{ Rhi-milR-89 } & LOC_Os06g33020.1 & 2.5 & $\begin{array}{l}\text { cDNA I retrotransposon protein, putative, Ty3-gypsy } \\
\text { subclass, expressed }\end{array}$ & Cleavage \\
\hline & LOC_Os06g32890.1 & 2.5 & $\begin{array}{l}\text { cDNA I retrotransposon protein, putative, Ty3-gypsy } \\
\text { subclass, expressed }\end{array}$ & Cleavage \\
\hline \multirow{3}{*}{ Rhi-milR-9 } & LOC_Os02g35820.2 & 2.5 & $\begin{array}{l}\text { cDNA I retrotransposon protein, putative, } \\
\text { unclassified, expressed }\end{array}$ & Cleavage \\
\hline & LOC_Os02g35820.1 & 2.5 & $\begin{array}{l}\text { cDNA I retrotransposon protein, putative, } \\
\text { unclassified, expressed }\end{array}$ & Cleavage \\
\hline & LOC_Os07g04230.1 & 2.5 & $\begin{array}{c}\text { cDNA I retrotransposon protein, putative, } \\
\text { unclassified, expressed }\end{array}$ & Cleavage \\
\hline Rhi-milR-97 & LOC_Os07g47950.1 & 2.5 & cDNA I expressed protein & Cleavage \\
\hline scaffold130_44481 & LOC_Os09g29390.1 & 2.5 & $\begin{array}{l}\text { cDNA I plastocyanin-like domain containing protein, } \\
\text { putative, expressed }\end{array}$ & Cleavage \\
\hline \multirow{2}{*}{ scaffold47_37559 } & LOC_Os04g52164.1 & 2 & $\begin{array}{l}\text { cDNA I transferase family protein, } \\
\text { putative, expressed }\end{array}$ & Cleavage \\
\hline & LOC_Os04g52164.2 & 2 & $\begin{array}{l}\text { cDNA I transferase family protein, } \\
\text { putative, expressed }\end{array}$ & Cleavage \\
\hline
\end{tabular}

\section{5. qRT-PCR Expression Analysis of milRNAs}

Seventeen milRNAs (known and novel) were selected based on read count, target genes in R. solani, and also based on the target genes in rice. The relative expression of fungal encoded milRNAs during host (rice) infection was analyzed at different time points of infection, different age and genetic makeup of host tissue, and also in different strains of fungi. The number of expressed milRNAs varied in different experiments, therefore, only those milRNA were included which showed expression in at least one sample. 


\subsubsection{At Different Time Points of Inoculation}

TN1, the susceptible rice cultivar, was inoculated with $R$. solani to study the expression levels of fungal-encoded milRNAs at different time points of infection. While comparing with the expression of milRNAs in $R$. solani grown in PDA medium, all the milRNAs except Rhi-milR-160 showed down-regulation during the rice infection at all time points. Furthermore, the milRNAs were differentially regulated at different time point of infection. Novel milRNAs scaffold130_44481 and scaffold47_37005, and known milRNAs Rhi-milR-35 and Rhi-milR-169 expressed at all time points, while scaffold7_15416 and Rhi-milR-160 expressed at all the time points except at $18 \mathrm{~h}$. Among all the milRNAs analyzed, only Rhi-milR-160 showed up-regulation (at $48 \mathrm{~h}$ and $72 \mathrm{~h}$ time points). Scaffold1_2221 expressed at $48 \mathrm{~h}, 72 \mathrm{~h}, 96 \mathrm{~h}$ and $5 \mathrm{~d}$ while scaffold47_37559 showed expression at 18 h, 24 h, and 5 days. Expression of Rhi-milR-22, Rhi-milR-43, Rhi-milR-120 and Rhi-milR-146 was observed exclusively at a specific time point of infection, i.e.,72 h, 5 days, $48 \mathrm{~h}$ and 5 days respectively. Notably, the Rhi-milR-43 showed down-regulation at 5 days. While analyzing the expression of milRNAs at different time points, most of the milRNAs showed expression at 5 days (Figure 2).
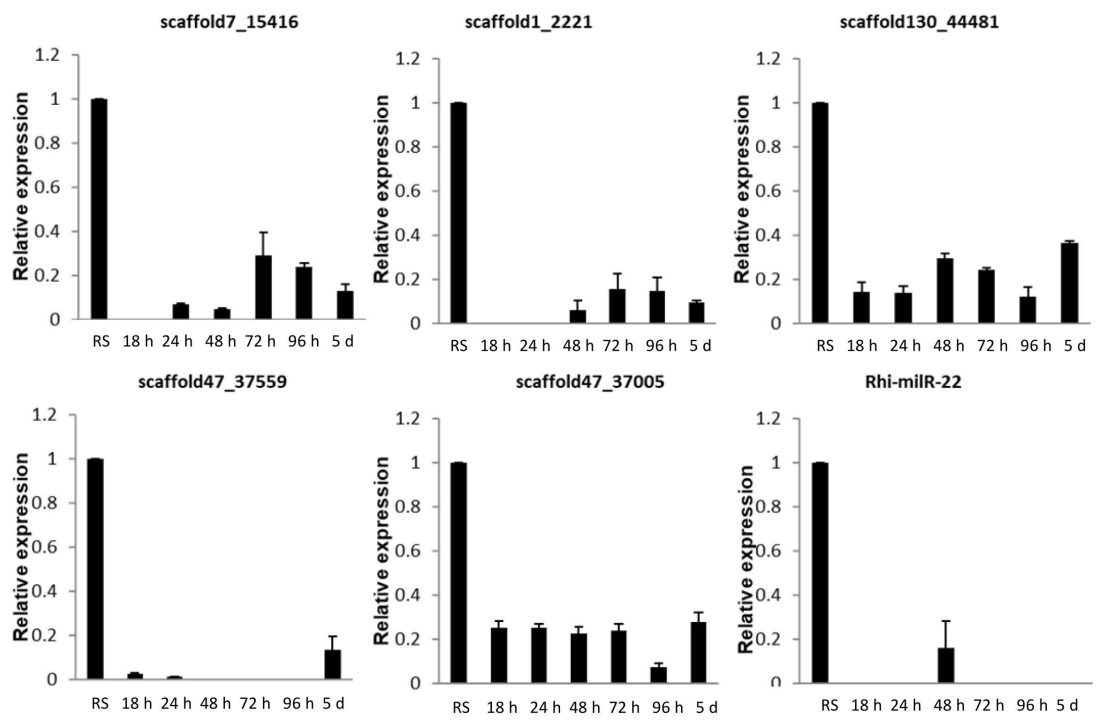

Rhi-milR-22
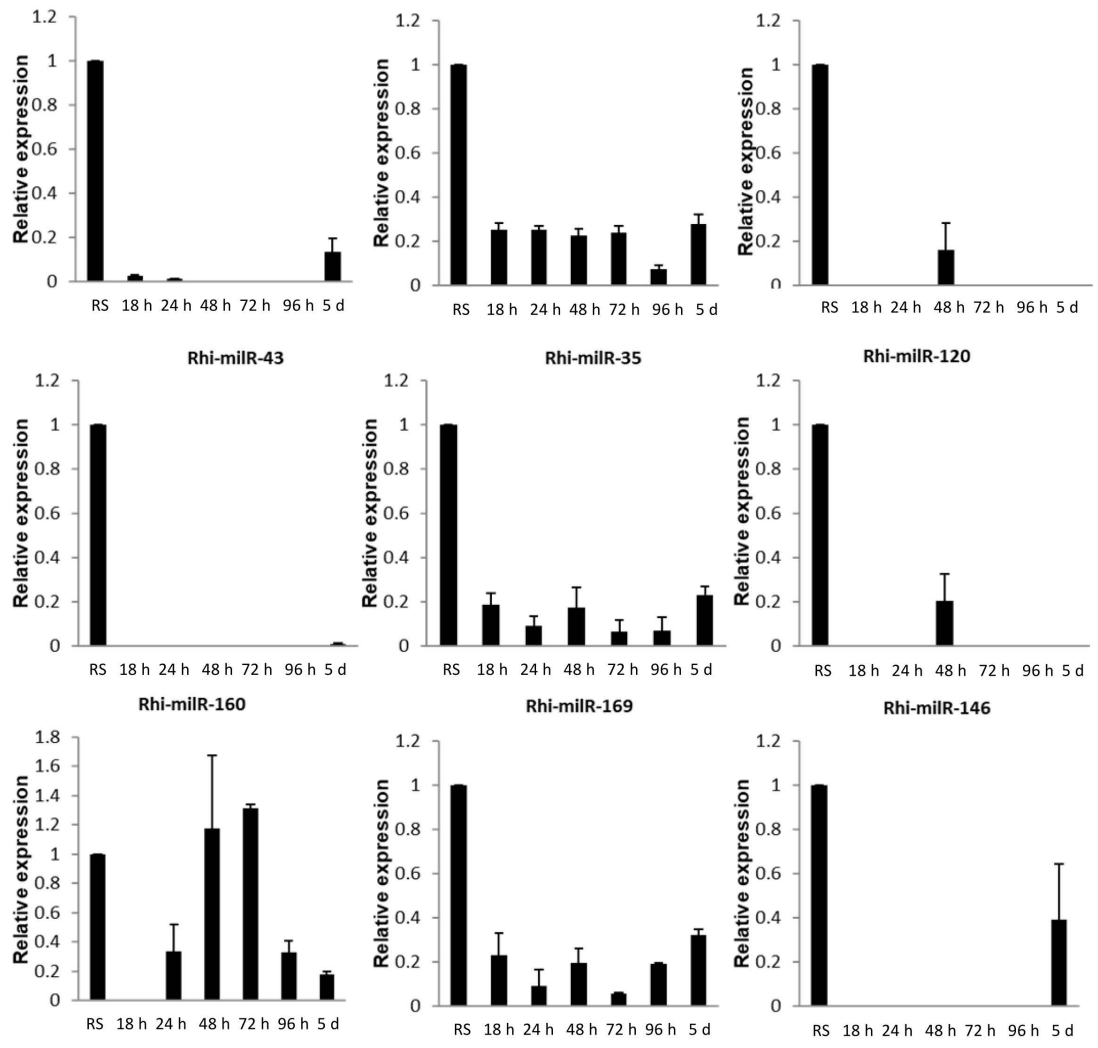

Figure 2. qRT-PCR expression analysis of milRNAs at different time points after fungal inoculation. X-axis: samples (RS: R. solani cultured in potato dextrose agar medium, $18 \mathrm{~h}$ to $5 \mathrm{~d}$ : $R$. solani inoculated sheath tissue of TN1 harvested at different time points); Y-axis: Expression level of the individual milRNA relative to its expression in $R$. solani grown in PDA. Error bars indicate the mean \pm S.E. of three biological replicates. 


\subsubsection{In Different Growth Stages of Host Plant}

The expression of fungal encoded milRNAs was influenced by the growth stage of its host. All the milRNAs except Rhi-milR-16 showed down-regulation at both the growth stages during infection when compared with the expression level of milRNAs in fungi grown in PDA medium. Among the two growth stages, scaffold7_15416, RhimilR-160, and Rhi-milR-169 showed more expression at reproductive stage (80 days old) of host than vegetative ( 45 days old). Rhi-milR- 43 showed down-regulation in a 45 -dayold host and the expression levels further declined in an 80-day-old host. Interestingly, Rhi-milR-16 did not show expression in a 45-day-old host but was up-regulated in an 80-day-old host; whereas Rhi-milR-146 did not show expression in 80 days old host but was down-regulated in a 45-day-old host. Scaffold1_2221 and Rhi-milR-135 also showed stage specific expression, i.e., down-regulation in 45 and 80-day-old hosts, respectively. Scaffold7_15416, scaffold130_44481, scaffold47_37559, scaffold47_37005, Rhi-milR-43, RhimilR-35, Rhi-milR-160, and Rhi-milR-169 showed down-regulation in both 45-dayold and 80-day-old hosts (Figure 3).
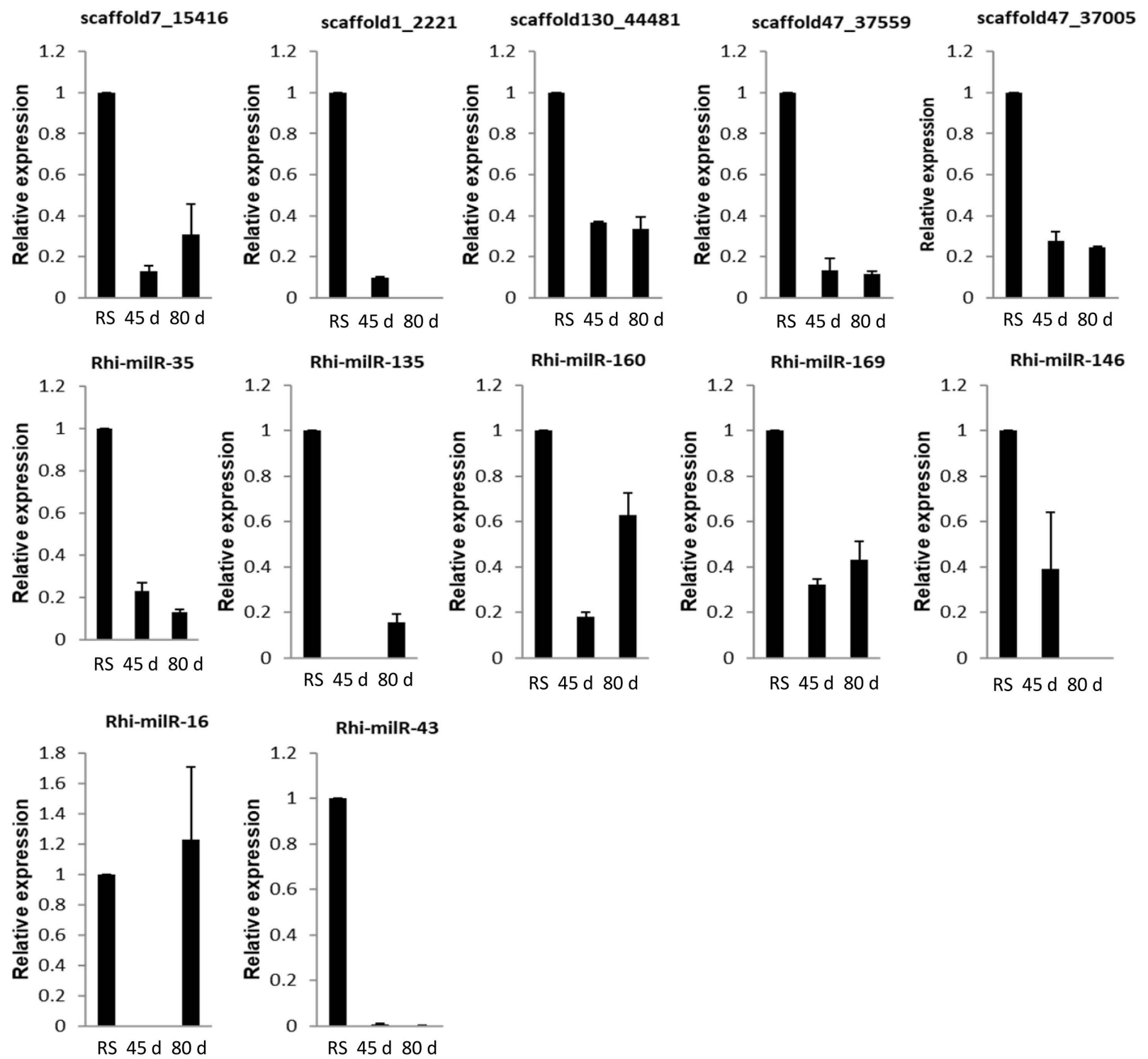

Figure 3. qRT-PCR expression analysis of milRNAs in sheath tissue of 45 days and 80 days old TN1 plants after R. solani inoculation. X-axis: samples; Y-axis: Expression level of the individual milRNA relative to its expression in $R$. solani grown in PDA. Error bars indicate the mean \pm standard error (SE) of three biological replicates. 


\subsubsection{In Different Rice Genotypes}

Expression of fungal-encoded milRNAs was analysed during the infection of four different genotypes of rice, i.e., Tetep, BPT5204, Pankaj, and TN1. Forty-five day-old plants of these genotypes were inoculated with Wgl-2 strain of $R$. solani and samples were collected after 5 days of inoculation. All the milRNAs except Rhi-milR-160 showed downregulation during infection of four rice genotypes when compared with the expression level of milRNAs in fungi grown in PDA medium. Seven milRNAs: scaffold7_15416, scaffold130_44481, scaffold47_37559, scaffold47_37005, Rhi-milR-35, Rhi-milR-160, and RhimilR-169 showed expression in all the genotypes. These milRNAs were down-regulated during infection of rice genotypes. Among these, expression of scaffold130_44481 was more in tolerant (Tetep and Pankaj) than the susceptible (TN1 and BPT5204) rice genotypes. Expression of scaffold47_37559 was greater in Pankaj than the other three genotypes while, Rhi-milR-169 expression was lesser in Pankaj than the other three genotypes. Rhi-milR-160 showed up-regulation in Tetep and BPT5204 but down-regulation in Pankaj and TN1. Rhi-milR-120 and Rhi-milR-135 showed exclusive expression in Pankaj while Rhi-milR-146 showed exclusive expression in TN1. Scaffold1_2221 expressed in all genotypes except BPT5204 (Figure 4).
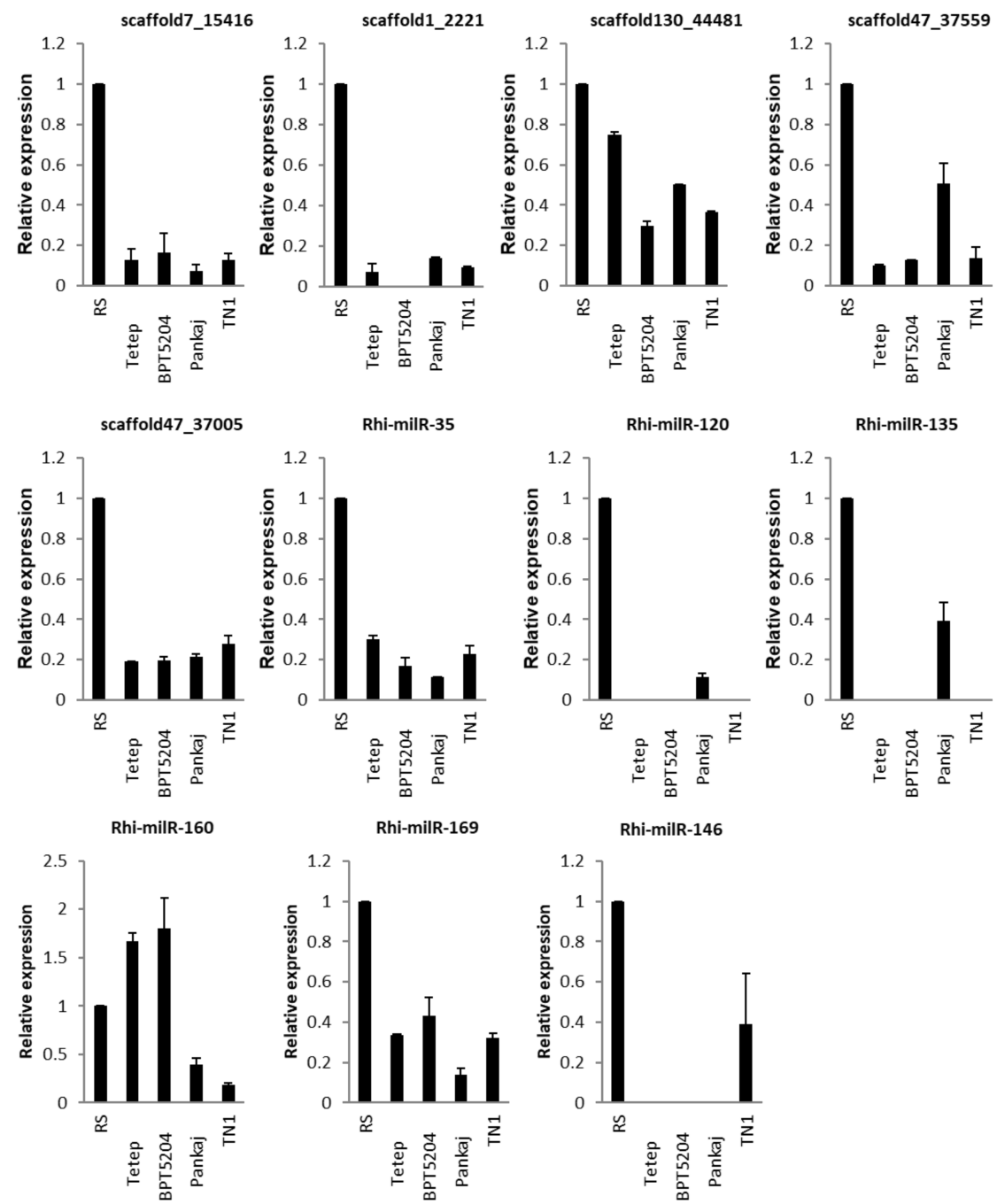

Figure 4. qRT-PCR expression analysis of milRNAs in different rice genotypes after $R$. solani inoculation. X-axis: samples (R. solani inoculated sheath tissue of four rice genotypes); Y-axis: Expression level of the individual milRNA relative to its expression in $R$. solani grown in PDA. Error bars indicate the mean \pm S.E. of three biological replicates. 


\subsubsection{In Different Strains of Fungus}

Expression of milRNAs of four different fungal strains was analysed during the infection of the TN1 rice genotype. All the milRNAs except scaffold1_2221 showed downregulation during infection in all fungal strains when compared with the expression level of respective milRNAs in fungi grown in PDA medium. Novel milRNAs scaffold7_15416, scaffold1_2221, scaffold130_44481, scaffold47_3559, scaffold47_37005 and known milRNAs Rhi-milR-35, Rhi-milR-160 and Rhi-milR-169 showed expression in all the four strains of fungi while infecting its host. Among these, scaffold7_15416, scaffold1_2221, scaffold47_37559, and Rhi-milR-160 showed more expression in Imph-2 strain than three other fungal strains. Furthermore, scaffold47_35943, scaffold47_36931, Rhi-milR-43, Rhi-milR-57, and Rhi-milR-135 showed expression only in Imph-2 strain while infecting TN1. Notably, scaffold1_2221 was up-regulated in Imph-2 strain but down-regulated in the other three strains. Rh-milR-16 and Rhi-milR-146 showed exclusive expression in Chn-1 and Wgl-2 strains, respectively. Rhi-milR-120 did not show expression in the Wgl-2 strain (Figure 5).
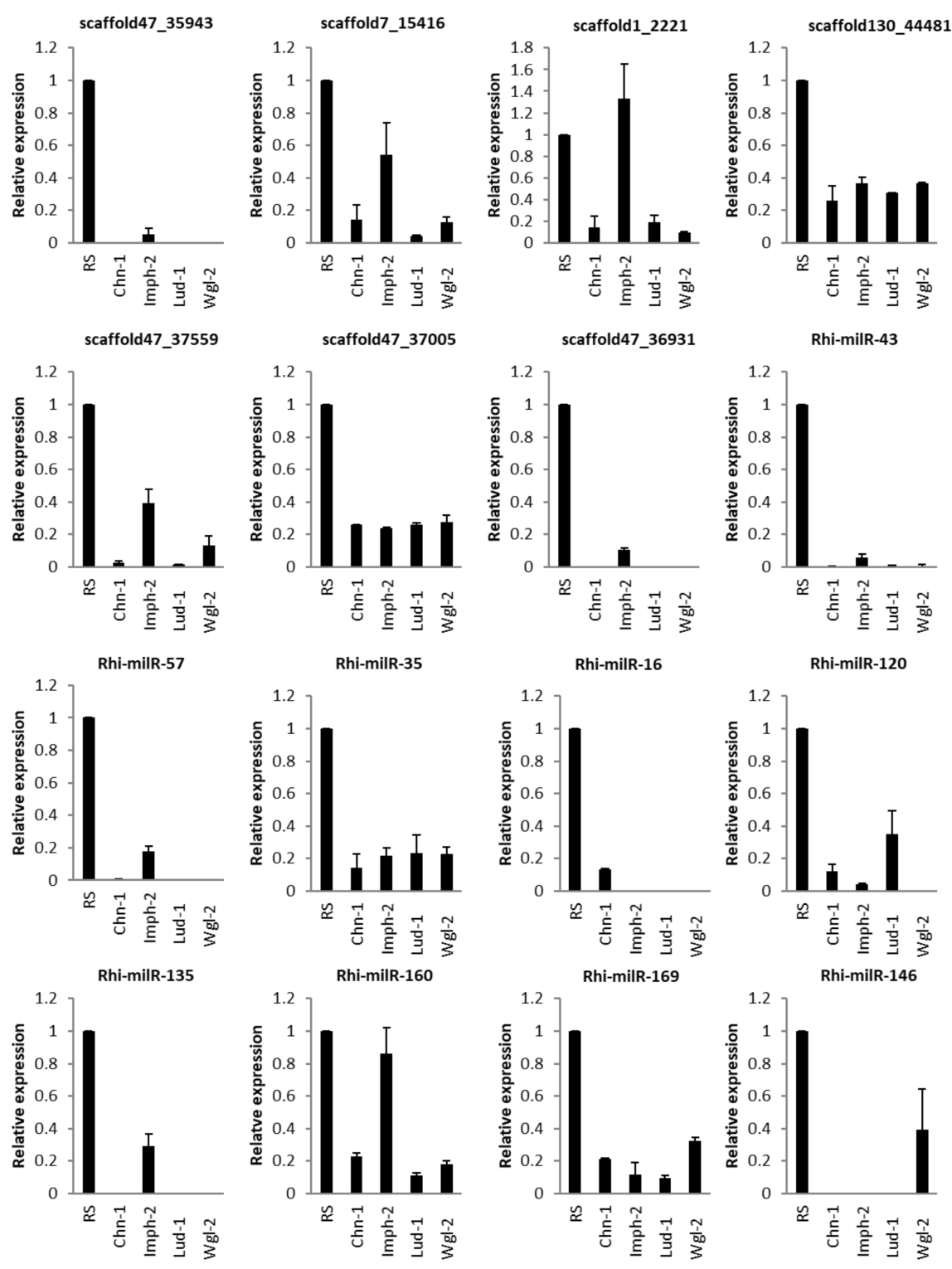

Figure 5. qRT-PCR expression analysis of milRNAs in sheath tissue of TN1 after inoculation with different strains of $R$. solani. X-axis: samples (sheath tissue of TN1 inoculated with four different strains of $R$. solani); Y-axis: Expression level of the individual milRNA relative to its expression in $R$. solani grown in PDA. Error bars indicate the mean \pm S.E. 


\subsubsection{Expression Analysis of Fungal and Plant Target Genes}

Expression of seven plant target genes and two fungal target genes was analysed in R. solani-infected rice (TN1) tissue. Along with the target genes, the expression of the respective regulatory milRNA was also analysed. Among the plant target genes, the regulons Osa-KANADI1:Rhi-milR-124, Osa-vacuolar-sorting receptor precursor:Rhi-milR-13, Osa-plastocyanin-like domain containing protein:Rhi-milR-22, and Osa-serine/threonineprotein kinase receptor precursor: Rhi-milR-51 showed anticipated reciprocal trend of expression. In these cases, the fungal encoded milRNAs and their respective plant target genes showed opposite trend of expression during the disease development (Figure 6a). Similarly, fungal genes AGA1IA_02889 and AGA1IA_07743 and their regulatory milRNAs Rhi-milR-141 and Rhi-milR-120 showed a contrasting expression pattern in infected tissue of rice (Figure $6 b$ ).

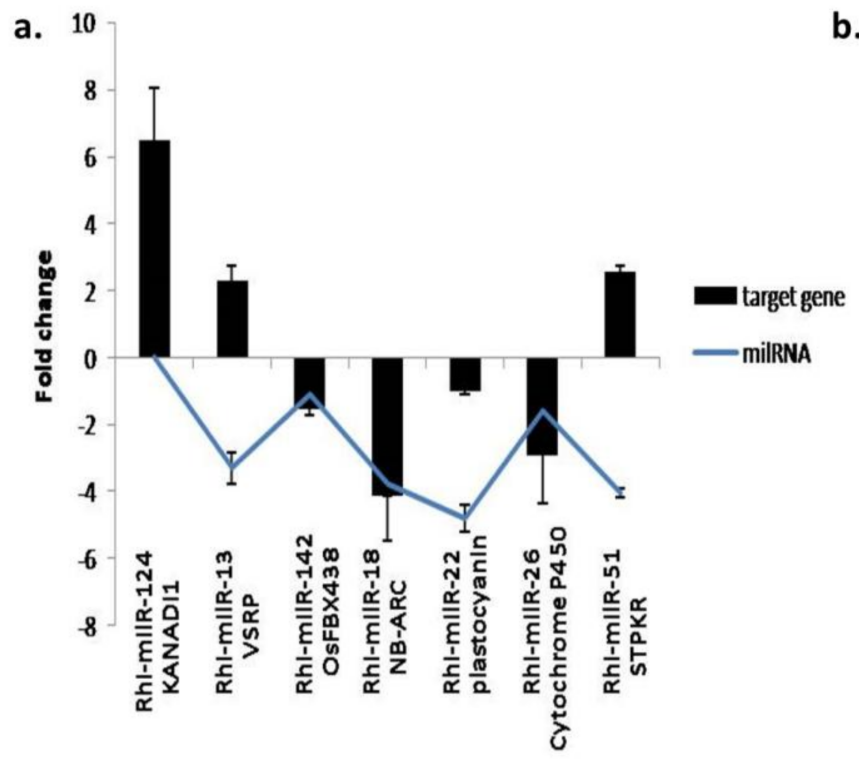

b.

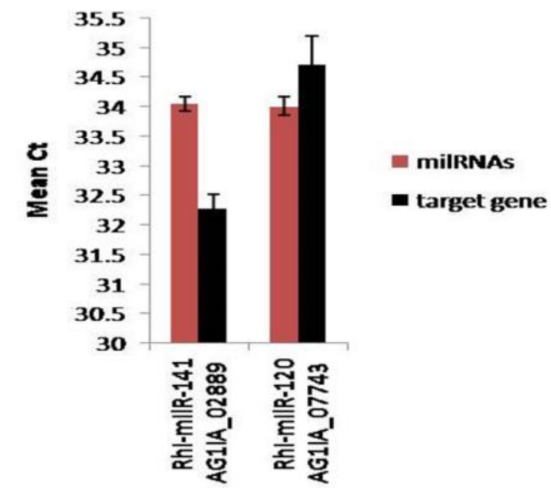

Figure 6. Expression analysis of target genes (a) qRT-PCR expression analysis of fungal milRNAs and their respective plant target genes. (b) qRT-PCR expression analysis of fungal milRNAs and their respective fungal target genes.

\section{Discussion}

MicroRNAs are regulatory genes that play critical roles in cellular processes of almost all eukaryotes [14]. It was believed that fungi do not possess microRNAs until the discovery of miRNA-like small RNAs (milRNAs) produced through at least four different biogenesis pathways in model fungi N. crassa [6,32]. Compared to plants and animals, there are very limited reports on milRNAs of phytopathogenic fungi which are primary agents causing diseases and huge loss to agriculture crops. In the absence of resistance in the rice germplasm against $R$. solani, it is extremely important to decipher the gene regulatory networks and biology of pathogens to devise effective strategies for controlling the menace of sheath blight disease in rice [31,33-35]. We analyzed the milRNAs of sheath blight disease causing pathogen $R$. solani AG1 IA by RNAseq of small RNAs. A total of 128 known and 22 novel milRNAs were identified. Most of the milRNAs showed low abundance which is accordance with earlier study of fungal milRNAs [36]. Notably, known milRNAs-Rhi-milR-16, Rhi-milR-22, Rhi-milR-35, Rhi-milR-57, Rhi-milR-91, and Rhi-milR-112 and novel milRNAs-scaffold13_22000, scaffold47_37005, scaffold47_37523, scaffold47_37559, scaffold47_37341, scaffold47_37407, and scaffold47_36418 showed more abundance than other microRNAs suggesting their significant role in fungal gene regulation. The novel milRNA scaffold13_22000 showed $>6000$ read count which emphasizes that several novel players identified in this study might regulate fungal genome. Earlier studies 
suggested that such novel milRNAs may have crucial roles in pathogenesis and virulence of $R$. solani [21,25]. 1725 target genes of 128 known and 22 novel milRNAs were identified that are involved in various metabolic and developmental processes of fungi indicating that milRNAs are major regulators of the $R$. solani genome. The role of milRNAs in fungal growth and development has been recently described in Sclerotinia sclerotiorum [37]. A number of milRNAs were identified in the soil-borne fungal pathogen Verticillium dahlia causing wilt diseases in several agriculture crops. One of the milRNAs (VdmilRNA1) was demonstrated to mediate epigenetic repression of a virulence gene (VdHy1) in pathogenic fungi [38]. Furthermore, the role of milRNAs (CmmilR4 and CmilR16) in fruiting body development was demonstrated in Cordyceps militaris [39]. The milRNAs-Rhi-milR-141, Rhi-milR-120, Rhi-milR-41, and Rhi-milR-91 target pectinesterase and pectate lyase domaincontaining protein genes signifying their crucial role in plant cell wall degradation and pathogenesis. milRNAs of $R$. solani targeting carbohydrate active enzymes and other fungal genes including those involved in virulence and pathogen-host interaction were reported recently [25]. The genes encoding plant cell wall degrading enzymes of $R$. solani play significant role in disease initiation and establishment [4]. Vm-milR16 of Valsamali regulates the expression of pectinase genes during $V$. Mali-host interaction [40].

Interestingly, R. solani milRNAs showed their targets in the host (rice) genome also. A similar observation was made on $R$. solani targeting maize genes [25]. Recently, cross kingdom regulation of genes by miRNAs during host-pathogen interaction has been demonstrated in several pathosystems [41]. Bidirectional cross-kingdom transport of microRNAs or small RNAs through naked form, combined with RNA-binding proteins or enclosed by vesicles between fungal pathogens and its host plants has been reported [42]. In an interesting observation, Cui et al. (2019) [43] demonstrated that bba-milR1 encoded by pathogenic fungus Beauveria bassiana hijacks the RNA-interference machinery of a host (mosquito) to attenuate host immunity and facilitate infection. The fungus Sclerotinia sclerotiorum was reported to produce at least 374 distinct highly abundant sRNAs during infection of Arabidopsis thaliana and Phaseolus vulgaris. Targets of these small RNAs were significantly down-regulated during infection in A. thaliana [44].

Rhi-milR-124 targets Osa-KANADI1. In Arabidopsis, KANADI1 acts as a transcriptional repressor of genes involved in auxin biosynthesis, auxin transport, and auxin response [45]. Fungal pathogens control the auxin levels and auxin signalling pathways that significantly influence the defense network in plants [46]. In the wheat-Zymoseptoria tritici pathosystem, Ma et al. [47] showed that as an immune response, the fungal infection induced wheat small RNAs that regulate auxin-related genes. Rhi-milR-13 regulates Osa-vacuolar-sorting receptor (VSR) of rice which is responsible for targeting defenserelated soluble proteins to the vacuole. Plant vacuoles play an important role in defense by releasing hydrolytic enzymes and antimicrobial compounds upon pathogen infection that leads to programmed cell death due to hypersensitive response [48]. Suppression of rice VSR by $R$. solani milRNA might suppress the plant defense by inhibiting the hypersensitive response. Notably, the target probability in the case of Osa-vacuolar-sorting receptor precursorRhi-milR-13 is very high with expectedvalue of 1.0. Therefore, further probing of this milRNA-target gene regulation network can add significant information on host-pathogen molecular cross-talk. Rhi-milR-131 targets nuclear transcription factor Y (NFY) subunit of rice which is also regulated by the host miRNA, i.e., Osa-miR169. The over-expression of Osa-miR169 in rice led to repression of NFY resulting in hypersusceptibility of transgenic rice against blast fungus Magnaportheoryzae [49]. Repression of NFY by Rhi-milR-131 may weaken the defense response of rice against $R$. solani. Other host target genes of fungal milRNAs included isoflavone reductase, OsFBX438-F-box domain containing protein, NB-ARC domain containing protein, serine/threonine-protein kinase receptor, and rabGAP/TBC domain-containing protein which are involved in plant defense against pathogens [50-54]. R. solani-encoded milRNAs target maize defensegenesduring infection and negatively regulate resistance of maize [25]. Notably, the regulons such as Osa-KANADI1:Rhi-milR-124, Osa-vacuolar-sorting receptor precursor:Rhi-milR-13, 
Osa-plastocyanin-like domain containing protein:Rhi-milR-22, and Osa-serine/threonineprotein kinase receptor precursor: Rhi-milR-51 were verified by analysing their expression through qRT-PCR during host-fungal interaction and disease development. Recent reports demonstrate a role of cross-kingdom RNAi in regulation of genes associated with pathogen virulence and host resistance. For example, small RNAs of phytopathogenic fungi Botrytis cinerea trigger the silencing of immunity-related genes of its hosts $A$. Thaliana and Solanum lycopersicum [55]. A similar observation has also recently been reported inthe case of Puccinia triticina and P. striiformis [56,57]. In addition to plant defense genes, fungal milRNAs showed their target as genes associated with growth and the development of the host.

This study provides a first comprehensive analysis of milRNAs expression during pathogen infection as the expression of 17 milRNAs was analysed at different time points of infection, at different growth stages of the host, in different genotypes (with different genetic make-up) of host, and also in different strains of fungi. The down-regulation of all milRNAs except Rhi-milR-160 was noticed at all the time points of infection suggesting the induction of their target genes during the disease initiation and establishment. The differential regulation of milRNAs at different time points of infection suggests that fungal genes are regulated by milRNAs during disease initiation and progression. Scaffold130_44481, scaffold47_37005, Rhi-milR-35, and Rhi-milR-169 showed expression throughout all time points of infection indicating their central role in infection. Expression of Rhi-milR-22, Rhi-milR-43, Rhi-milR-120, and Rhi-milR-146 was observed specifically at $72 \mathrm{~h}, 5 \mathrm{~d}, 48 \mathrm{~h}$, and $5 \mathrm{~d}$ respectively, suggesting that fungi recruit specific milRNAs as the infection progresses. During the infection and disease progression, $R$. solani requires mycotoxin production and penetration of mycelium inside the host cells. Studies in Aspergillus flavus and Fusarium oxysporum $\mathrm{f}$. sp. Niveum highlighted the significant roles of milRNAs in the mycelium growth and mycotoxin biosynthesis [22,58]. Lin et al. (2016) [21] analysed the differential expression pattern of $R$. solani milRNAs during infection and suggested their roles in disease initiation and establishment [58]. Differential regulation of milRNAs during sclerotial development in Sclerotinia sclerotiorum was observed by analyzing small RNAs at different time points [59].

At the time of infection, expression of scaffold7_15416, Rhi-milR-160, and Rhi-milR169 was more at the reproductive stage than vegetative stage of rice while Rhi-milR-16 did not show expression in vegetative stage but was up-regulated in the reproductive stage. By contrast, Rhi-milR-146 was down-regulated in the vegetative stage but no expression was observed in the reproductive stage. These results suggest that growth stage of host plant influences the milRNAs expression of fungi. Scaffold1_2221 was down-regulated in the vegetative stage while Rhi-milR-135 was down-regulated in reproductive stage, indicating their specific role during pathogenesis of different-aged hosts. Scaffold7_15416, scaffold130_44481, scaffold47_37559, scaffold47_37005, Rhi-milR-43, Rhi-milR-35, Rhi-milR160, and Rhi-milR-169 were down-regulated at both the growth stages signifying their essential roles during infection.

Expression of seven-milRNAs-scaffold7_15416, scaffold130_44481, scaffold47_37559, scaffold47_37005, Rhi-milR-35, Rhi-milR-160, and Rhi-milR-169 was down-regulated while infecting the four rice genotypes suggesting their key roles in regulating the genes during infection. Expression of scaffold130_44481 and scaffold47_37559 was greater in tolerant genotypes than susceptible rice genotypes while Rhi-milR-120 and Rhi-milR-135 showed exclusive expression in Pankaj indicating that regulation by milRNAs is significantly influenced by host genetic architecture. Expression of other milRNAs also varied in different genotypes of its host.

Expression of scaffold7_15416, scaffold1_2221, scaffold130_44481, scaffold47_3559, scaffold47_37005, Rhi-milR-35, Rhi-milR-160, and Rhi-milR-169 was observed in all the four strains of fungi while infecting its host suggesting their primary role in infection of the host. Notably, expression of scaffold7_15416, scaffold1_2221, scaffold47_37559, and Rhi-milR-160 was greater in Imph-2 strain while scaffold47_35943, scaffold47_36931, 
Rhi-milR-43, Rhi-milR-57, and Rhi-milR-135 were exclusively expressed in Imph-2 strain indicating that different strains of a fungus have evolved differential milRNA mediated gene regulation. Imph-2 and Chn-1 are mild strains of R. solani [26]. Rh-milR-16 showed exclusive expression in Chn-1 while Rhi-milR-146 showed exclusive expression in the Wgl-2 strain. Expression pattern of milRNAs in this study indicate that milRNAs may play critical role in determining the virulence of fungal strains. Vm-milR16 of the phytopathogenic fungus $V$. mali regulates the expression of virulence genes [40].

Fungal genome-encoded microRNA-like RNAs are relatively less explored genetic elements that play a major role in regulation and expression of genes associated with growth, development, pathogenesis, and virulence. We made a comprehensive effort by combining RNAseq and qRT-PCR assays to decipher the milRNAs of an Indian strain of $R$. solani AG1-1A causing sheath blight disease of rice. Several milRNA candidates targeting key genes of $R$. solani were identified which need to be further characterized to understand their specific biological roles. The novel milRNAs are significant additions to understanding of the complexity of gene regulation of $R$. solani. The novel milRNA scaffold13_22000 showing very high expression needs to be characterized for its role in pathogen-host interaction and fungal metabolism. Further probing the regulation of rice genes by fungal milRNAs will be a milestone in understanding the molecular basis of host-pathogen interaction. Some of the gene regulatory networks such as Osa-KANADI1:Rhi-milR-124, Osa-vacuolar-sorting receptor precursor:Rhi-milR-13, and Osa-nuclear transcription factor Y:Rhi-milR-131 can be studied on priority. Also, the better understanding regulation of rice defense genes by $R$. solani milRNAs may help in executing more effective strategies against this deadly plant pathogen. Detailed expression analysis of milRNAs at different time points of infection, at different growth stages of hosts, in different genotypes of hosts, and in different strains of fungi provide ample information to plan future studies for understanding the gene regulation mechanism of $R$. solani. Our results indicate that milRNAs may contribute to almost all the metabolic processes of the $R$. solani pathogen.

Supplementary Materials: The following are available online at https://www.mdpi.com/article/ 10.3390/jof7070561/s1, Table S1: List of primers used in this study for qRT-PCR assays. Table S2: List of known milRNAs identified in this study. Table S3: List of target genes of known and novel milRNAs. Table S4: Gene ontology analysis of selected target genes.

Author Contributions: Conceived and designed the experiments: S.K.M. Performed the experiments: N.B.P. and S.K.M. Analysis of data: S.K.M., N.B.P., S.K.S., S.N. Wrote the article: S.K.M., N.B.P., S.M.B., C.V.D.R., R.M.S. Provided the resources: V.P., Y.C.M. and R.M.S. All authors have read and agreed to the published version of the manuscript.

Funding: Financial support was received from Department of Biotechnology (DBT), Government of India (GrantNo. BT/PR6466/COE/34/16/2012).

Institutional Review Board Statement: Not applicable.

Informed Consent Statement: Not applicable.

Data Availability Statement: All data generated or analysed during this study are included in this published article (and its Supplementary Information files). The sequence data is available on request.

Acknowledgments: Authors are thankful to the Director, ICAR-IIRR, for his kind support. Financial support received from DBT Grant (BT/PR6466/COE/34/16/2012) is acknowledged. NBP thanks PJTSAU, Hyderabad for providing fellowship. We are thankful to Nucleome Informatics Pvt. Ltd., Hyderabad, India for providing the commercial facility of RNA sequencing. Authors thank G.S. Laha for providing the fungal strains.

Conflicts of Interest: The authors declare no competing interest. 


\section{References}

1. Bernardes-De-Assis, J.; Storari, M.; Zala, M.; Wang, W.; Jiang, D.; Shidong, L.; Jin, M.; McDonald, B.; Ceresini, P. Genetic Structure of Populations of the Rice-Infecting Pathogen Rhizoctonia solani AG-1 IA from China. Phytopathology 2009, 99, 1090-1099. [CrossRef]

2. Boukaew, S.; Prasertsan, P. Suppression of rice sheath blight disease using a heat stable culture filtrate from Streptomyces philanthi RM-1-138. Crop Prot. 2014, 61, 1-10. [CrossRef]

3. Meyers, B.C.; Axtell, M.J.; Bartel, B.; Bartel, D.P.; Baulcombe, D.; Bowman, J.L.; Cao, X.; Carrington, J.C.; Chen, X.; Green, P.J.; et al. Criteria for Annotation of Plant MicroRNAs. Plant Cell 2008, 20, 3186-3190. [CrossRef]

4. Rao, T.B.; Chopperla, R.; Methre, R.; Punniakotti, E.; Venkatesh, V.; Sailaja, B.; Raghurami Reddy, M.; Yugander, A.; Laha, G.S.; SheshuMadhav, M.; et al. Pectin induced transcriptome of a Rhizoctonia solani strain causing sheath blight disease in rice reveals insights on key genes and RNAi machinery for development of pathogen derived resistance. Plant Mol. Biol. 2019, $100,59-71$. [CrossRef] [PubMed]

5. Zheng, A.; Lin, R.; Zhang, D.; Qin, P.; Xu, L.; Ai, P.; Ding, L.; Wang, Y.; Chen, Y.; Liu, Y.; et al. The evolution and pathogenic mechanisms of the rice sheath blight pathogen. Nat. Commun. 2013, 4, 1424. [CrossRef]

6. Lee, B.-M.; Park, Y.-J.; Park, D.-S.; Kang, H.-W.; Kim, J.-H.; Song, E.-S.; Park, I.-C.; Yoon, U.-H.; Hahn, J.-H.; Koo, B.-S.; et al. The genome sequence of Xanthomonas oryzae pathovar oryzae KACC10331, the bacterial blight pathogen of rice. Nucleic Acids Res. 2005, 33, 577-586. [CrossRef]

7. Mücke, S.; Reschke, M.; Erkes, A.; Schwietzer, C.-A.; Becker, S.; Streubel, J.; Morgan, R.D.; Wilson, G.G.; Grau, J.; Boch, J. Transcriptional Reprogramming of Rice Cells by Xanthomonas oryzae TALEs. Front. Plant Sci. 2019, 10, 162. [CrossRef]

8. Oliva, R.; Ji, C.; Atienza-Grande, G.; Huguet-Tapia, J.C.; Perez-Quintero, A.; Li, T.; Eom, J.-S.; Li, C.; Nguyen, H.; Liu, B.; et al. Broad-spectrum resistance to bacterial blight in rice using genome editing. Nat. Biotechnol. 2019, 37, 1344-1350. [CrossRef]

9. Sundaram, R.M.; Vishnupriya, M.R.; Biradar, S.K.; Laha, G.S.; Reddy, G.A.; Rani, N.S.; Sarma, N.P.; Sonti, R.V. Marker assisted introgression of bacterial blight resistance in Samba Mahsuri, an elite indica rice variety. Euphytica 2008, 160, 411-422. [CrossRef]

10. Rampersad, S.N. Pathogenomics and Management of Fusarium Diseases in Plants. Pathogens 2020, 9, 340. [CrossRef]

11. Dong, O.X.; Ronald, P.C. Genetic Engineering for Disease Resistance in Plants: Recent Progress and Future Perspectives. Plant Physiol. 2019, 180, 26-38. [CrossRef]

12. Cools, H.J.; Hammond-Kosack, K. Exploitation of genomics in fungicide research: Current status and future perspectives. Mol. Plant Pathol. 2012, 14, 197-210. [CrossRef]

13. Bhogireddy, S.; Mangrauthia, S.K.; Kumar, R.; Pandey, A.K.; Singh, S.; Jain, A.; Budak, H.; Varshney, R.K.; Kudapa, H. Regulatory non-coding RNAs: A new frontier in regulation of plant biology. Funct. Integr. Genom. 2021, 1-18. [CrossRef]

14. Wani, S.H.; Kumar, V.; Khare, T.; Tripathi, P.; Shah, T.; Ramakrishna, C.; Aglawe, S.; Mangrauthia, S.K. miRNA applications for engineering abiotic stress tolerance in plants. Biologia 2020, 75, 1063-1081. [CrossRef]

15. Vazquez, F.; Vaucheret, H.; Rajagopalan, R.; Lepers, C.; Gasciolli, V.; Mallory, A.C.; Hilbert, J.-L.; Bartel, D.P.; Crété, P. Endogenous trans-Acting siRNAs Regulate the Accumulation of Arabidopsis mRNAs. Mol. Cell 2004, 16, 69-79. [CrossRef] [PubMed]

16. Reinhart, B.J.; Bartel, D.P. Small RNAs Correspond to Centromere Heterochromatic Repeats. Science 2002, 297, 1831. [CrossRef]

17. Zilberman, D.; Cao, X.; Jacobsen, S.E. ARGONAUTE4 Control of Locus-Specific siRNA Accumulation and DNA and Histone Methylation. Science 2003, 299, 716-719. [CrossRef] [PubMed]

18. Taverna, S.D.; Coyne, R.S.; Allis, C.D. Methylation of Histone H3 at Lysine 9 Targets Programmed DNA Elimination in Tetrahymena. Cell 2002, 110, 701-711. [CrossRef]

19. Chen, R.; Jiang, N.; Jiang, Q.; Sun, X.; Wang, Y.; Zhang, H.; Hu, Z. Exploring MicroRNA-Like Small RNAs in the Filamentous Fungus Fusarium oxysporum. PLoS ONE 2014, 9, e104956. [CrossRef] [PubMed]

20. Chen, L.; Heikkinen, L.; Wang, C.; Yang, Y.; Sun, H.; Wong, G. Trends in the development of miRNA bioinformatics tools. Brief. Bioinform. 2019, 20, 1836-1852. [CrossRef] [PubMed]

21. Lin, R.; He, L.; He, J.; Qin, P.; Wang, Y.; Deng, Q.; Yang, X.; Li, S.; Wang, S.; Wang, W.; et al. Comprehensive analysis of microRNA-Seq and target mRNAs of rice sheath blight pathogen provides new insights into pathogenic regulatory mechanisms. DNA Res. 2016, 23, 415-425. [CrossRef] [PubMed]

22. Bai, Y.; Lan, F.; Yang, W.; Zhang, F.; Yang, K.; Li, Z.; Gao, P.; Wang, S. sRNA profiling in Aspergillus flavus reveals differentially expressed miRNA-like RNAs response to water activity and temperature. Fungal Genet. Biol. 2015, 81, 113-119. [CrossRef] [PubMed]

23. Yang, F. Genome-wide analysis of small RNAs in the wheat pathogenic fungus Zymoseptoria tritici. Fungal Biol. 2015, 119, 631-640. [CrossRef]

24. Lee, H.-C.; Li, L.; Gu, W.; Xue, Z.; Crosthwaite, S.K.; Pertsemlidis, A.; Lewis, Z.; Freitag, M.; Selker, E.U.; Mello, C.C.; et al. Diverse Pathways Generate MicroRNA-like RNAs and Dicer-Independent Small Interfering RNAs in Fungi. Mol. Cell 2010, $38,803-814$. [CrossRef] [PubMed]

25. Meng, H.; Wang, S.; Yang, W.; Ding, X.; Li, N.; Chu, Z.; Li, X. Identification of virulence associated milRNAs and their bidirectional targets in Rhizoctonia solani and maize during infection. BMC Plant Biol. 2021, 21, 155. [CrossRef]

26. Yugander, A.; Ladhalakshmi, D.; Prakasham, V.; Mangrauthia, S.K.; Prasad, M.S.; Krishnaveni, D.; Madhav, M.S.; Sundaram, R.M.; Laha, G.S. Pathogenic and Genetic Variation among the Isolates of Rhizoctonia solani (AG 1-IA), the Rice Sheath Blight Pathogen. J. Phytopathol. 2015, 163, 465-474. [CrossRef] 
27. Chen, L.; Ai, P.; Zhang, J.; Deng, Q.; Wang, S.; Li, S.; Zhu, J.; Li, P.; Zheng, A. RSIADB, a collective resource for genome and transcriptome analyses in Rhizoctonia solani AG1 IA. Database 2016, 2016, baw031. [CrossRef]

28. Axtell, M.J.; Meyers, B.C. Revisiting Criteria for Plant MicroRNA Annotation in the Era of Big Data. Plant Cell 2018, 30, $272-284$. [CrossRef] [PubMed]

29. Dai, X.; Zhuang, Z.; Zhao, P.X. psRNATarget: A plant small RNA target analysis server (2017 release). Nucleic Acids Res. 2018, 46, W49-W54. [CrossRef]

30. Mangrauthia, S.K.; Bhogireddy, S.; Agarwal, S.; Prasanth, V.V.; Voleti, S.R.; Neelamraju, S.; Subrahmanyam, D. Genome-wide changes in microRNA expression during short and prolonged heat stress and recovery in contrasting rice cultivars. J. Exp. Bot. 2017, 68, 2399-2412. [CrossRef] [PubMed]

31. Rao, T.B.; Chopperla, R.; Prathi, N.B.; Balakrishnan, M.; Prakasam, V.; Laha, G.S.; Balachandran, S.M.; Mangrauthia, S.K. A Comprehensive Gene Expression Profile of Pectin Degradation Enzymes Reveals the Molecular Events during Cell Wall Degradation and Pathogenesis of Rice Sheath Blight Pathogen Rhizoctonia solani AG1-IA. J. Fungi 2020, 6, 71. [CrossRef]

32. Chang, S.-S.; Zhang, Z.; Liu, Y. RNA Interference Pathways in Fungi: Mechanisms and Functions. Annu. Rev. Microbiol. 2012, 66, 305-323. [CrossRef] [PubMed]

33. Molla, K.A.; Karmakar, S.; Molla, J.; Bajaj, P.; Varshney, R.K.; Datta, S.K.; Datta, K. Understanding sheath blight resistance in rice: The road behind and the road ahead. Plant Biotechnol. J. 2020, 18, 895-915. [CrossRef] [PubMed]

34. Wang, A.; Shu, X.; Niu, X.; Zhao, W.; Ai, P.; Li, P.; Zheng, A. Comparison of gene co-networks analysis provide a systems view of rice (Oryza sativa L.) response to Tilletia horrida infection. PLoS ONE 2018, 13, e0202309. [CrossRef] [PubMed]

35. Zhang, J.; Zhao, W.; Fu, R.; Fu, C.; Wang, L.; Liu, H.; Li, S.; Deng, Q.; Wang, S.; Zhu, J.; et al. Comparison of gene co-networks reveals the molecular mechanisms of the rice (Oryza sativa L.) response to Rhizoctonia solani AG1 IA infection. Funct. Integr. Genom. 2018, 18, 545-557. [CrossRef] [PubMed]

36. Wang, L.; Xu, X.; Yang, J.; Chen, L.; Liu, B.; Liu, T.; Jin, Q. Integrated microRNA and mRNA analysis in the pathogenic filamentous fungus Trichophyton rubrum. BMC Genom. 2018, 19, 933. [CrossRef] [PubMed]

37. Xia, Z.; Wang, Z.; Kav, N.N.; Ding, C.; Liang, Y. Characterization of microRNA-like RNAs associated with sclerotial development in Sclerotinia sclerotiorum. Fungal Genet. Biol. 2020, 144, 103471. [CrossRef] [PubMed]

38. Jin, Y.; Zhao, J.-H.; Zhao, P.; Zhang, T.; Wang, S.; Guo, H.-S. A fungal milRNA mediates epigenetic repression of a virulence gene in Verticillium dahliae. Philos. Trans. R. Soc. B 2019, 374, 20180309. [CrossRef]

39. Shao, Y.; Tang, J.; Chen, S.; Wu, Y.; Wang, K.; Ma, B.; Zhou, Q.; Chen, A.; Wang, Y. milR4 and milR16 Mediated Fruiting Body Development in the Medicinal Fungus Cordyceps militaris. Front. Microbiol. 2019, 10, 83. [CrossRef] [PubMed]

40. Xu, M.; Guo, Y.; Tian, R.; Gao, C.; Guo, F.; Voegele, R.T.; Bao, J.; Li, C.; Jia, C.; Feng, H.; et al. Adaptive regulation of virulence genes by microRNA-like RNAs in Valsamali. New Phytol. 2020, 227, 899-913. [CrossRef]

41. Mathur, M.; Nair, A.; Kadoo, N. Plant-pathogen interactions: MicroRNA-mediated trans-kingdom gene regulation in fungi and their host plants. Genomics 2020, 112, 3021-3035. [CrossRef]

42. Wang, M.; Dean, R.A. Movement of small RNAs in and between plants and fungi. Mol. Plant Pathol. 2020, 21, 589-601. [CrossRef]

43. Cui, C.; Wang, Y.; Liu, J.; Zhao, J.; Sun, P.; Wang, S. A fungal pathogen deploys a small silencing RNA that attenuates mosquito immunity and facilitates infection. Nat. Commun. 2019, 10, 4298. [CrossRef]

44. Derbyshire, M.; Mbengue, M.; Barascud, M.; Navaud, O.; Raffaele, S. Small RNAs from the plant pathogenic fungus Sclerotinia sclerotiorum highlight host candidate genes associated with quantitative disease resistance. Mol. Plant Pathol. 2019, 20, $1279-1297$. [CrossRef] [PubMed]

45. Huang, T.; Harrar, Y.; Lin, C.; Reinhart, B.; Newell, N.R.; Talavera-Rauh, F.; Hokin, S.A.; Barton, M.K.; Kerstetter, R.A. Arabidopsis KANADI1 Acts as a Transcriptional Repressor by Interacting with a Specific cis-Element and Regulates Auxin Biosynthesis, Transport, and Signaling in Opposition to HD-ZIPIII Factors. Plant Cell 2014, 26, 246-262. [CrossRef]

46. Ludwig-Müller, J. Bacteria and fungi controlling plant growth by manipulating auxin: Balance between development and defense. J. Plant Physiol. 2015, 172, 4-12. [CrossRef] [PubMed]

47. Ma, X.; Wiedmer, J.; Palma-Guerrero, J. Small RNA Bidirectional Crosstalk During the Interaction Between Wheat and Zymoseptoria tritici. Front. Plant Sci. 2020, 10, 1669. [CrossRef]

48. Ruano, G.; Scheuring, D. Plant Cells under Attack: Unconventional Endomembrane Trafficking during Plant Defense. Plants 2020, 9, 389. [CrossRef] [PubMed]

49. Li, Y.; Zhao, S.-L.; Li, J.-L.; Hu, X.-H.; Wang, H.; Cao, X.-L.; Xu, Y.-J.; Zhao, Z.; Xiao, Z.-Y.; Yang, N.; et al. Osa-miR169 Negatively Regulates Rice Immunity against the Blast Fungus Magnaporthe oryzae. Front. Plant Sci. 2017, 8, 2. [CrossRef]

50. Cheng, Q.; Li, N.; Dong, L.; Zhang, D.; Fan, S.; Jiang, L.; Wang, X.; Xu, P.; Zhang, S. Overexpression of Soybean Isoflavone Reductase (GmIFR) Enhances Resistance to Phytophthora sojae in Soybean. Front. Plant Sci. 2015, 6, 1024. [CrossRef] [PubMed]

51. Feng, H.; Zhang, Q.; Wang, B.; Fu, Y.; Huang, L.; Wang, X.; Kang, Z. Exploration of microRNAs and their targets engaging in the resistance interaction between wheat and stripe rust. Front. Plant Sci. 2015, 6, 469. [CrossRef]

52. Goff, K.E.; Ramonell, K.M. The role and regulation of receptor-like kinases in plant defense. Gene Regul. Syst. Biol. 2007, 1, 167-175. [CrossRef]

53. van den Burg, H.A.; Tsitsigiannis, D.I.; Rowland, O.; Lo, J.; Rallapalli, G.; MacLean, D.; Takken, F.L.W.; Jones, J.D.G. The F-Box Protein ACRE189/ACIF1 Regulates Cell Death and Defense Responses Activated during Pathogen Recognition in Tobacco and Tomato. Plant Cell 2008, 20, 697-719. [CrossRef] 
54. Wen, Z.; Yao, L.; Wan, R.; Li, Z.; Liu, C.; Wang, X. Ectopic Expression in Arabidopsis thaliana of an NB-ARC Encoding Putative Disease Resistance Gene from Wild Chinese Vitis pseudoreticulata Enhances Resistance to Phytopathogenic Fungi and Bacteria. Front. Plant Sci. 2015, 6, 1087. [CrossRef]

55. Weiberg, A.; Wang, M.; Lin, F.-M.; Zhao, H.; Zhang, Z.; Kaloshian, I.; Huang, H.-D.; Jin, H. Fungal Small RNAs Suppress Plant Immunity by Hijacking Host RNA Interference Pathways. Science 2013, 342, 118-123. [CrossRef] [PubMed]

56. Dubey, H.; Kiran, K.; Jaswal, R.; Jain, P.; Kayastha, A.M.; Bhardwaj, S.C.; Mondal, T.K.; Sharma, T.R. Discovery and profiling of small RNAs from Puccinia triticina by deep sequencing and identification of their potential targets in wheat. Funct. Integr. Genom. 2019, 19, 391-407. [CrossRef] [PubMed]

57. Wang, B.; Sun, Y.; Song, N.; Zhao, M.; Liu, R.; Feng, H.; Wang, X.; Kang, Z. Puccinia striiformis f. sp. tritici mi croRNA -like RNA 1 (Pst -milR1), an important pathogenicity factor of Pst, impairs wheat resistance to Pst by suppressing the wheat pathogenesis-related 2 gene. New Phytol. 2017, 215, 338-350. [CrossRef] [PubMed]

58. Jiang, X.; Qiao, F.; Long, Y.; Cong, H.; Sun, H. MicroRNA-like RNAs in plant pathogenic fungus Fusarium oxysporum f. sp. niveum are involved in toxin gene expression fine tuning. 3 Biotech 2017, 7, 354. [CrossRef]

59. Zhou, J.; Fu, Y.; Xie, J.; Li, B.; Jiang, D.; Li, G.; Cheng, J. Identification of microRNA-like RNAs in a plant pathogenic fungus Sclerotinia sclerotiorum by high-throughput sequencing. Mol. Genet. Genom. 2012, 287, 275-282. [CrossRef] 